\title{
Deformation and breakup of a compound droplet in three-dimensional oscillatory shear flow
}

\author{
Haihu Liu ${ }^{a, *}$, Yang $\mathrm{Lu}^{\mathrm{a}}$, Sheng $\mathrm{Li}^{\mathrm{a}}$, Yuan Yu ${ }^{\mathrm{b}}$, Kirti Chandra Sahu ${ }^{\mathrm{c}}$ \\ a School of Energy and Power Engineering, Xi'an Jiaotong University, 28 West Xianning Road, Xi'an 710049, China \\ ${ }^{\mathrm{b}}$ School of Engineering, Sun Yat-Sen University, Guangzhou 510006, China \\ ${ }^{\mathrm{c}}$ Department of Chemical Engineering, Indian Institute of Technology Hyderabad Sangareddy, Telangana, 502 285, India
}

\section{A R T I C L E I N F O}

\section{Article history:}

Received 2 April 2020

Revised 5 September 2020

Accepted 20 September 2020

Available online 28 September 2020

\section{Keywords:}

Deformation and breakup

Compound droplet

Oscillatory shear

Wall confinement

Critical capillary number

\begin{abstract}
A B S T R A C T
A compound droplet subject to three-dimensional oscillatory shear flow is studied using a three-phase lattice Boltzmann model. Firstly, focusing on low values of capillary number $(\mathrm{Ca})$ where the compound droplet eventually reaches steady-state oscillatory condition, we study the effect of oscillatory period, viscosities of inner and outer fluids of the compound droplet, wall confinement and $\mathrm{Ca}$ on the droplet behavior. As the oscillatory period increases, the maximum deformation parameters gradually approach the steady-state values in the corresponding simple shear flow for both inner and outer droplets, and the compound droplet is more synchronous with applied shear. We demonstrate for the first time that due to high pressure near two tips inside the outer droplet the inner droplet may rotate counterintuitively in a direction opposite to the outer one. The compound droplet undergoes larger deformation when either droplet is less viscous, which also decreases the synchronization between inner and outer droplets. Increasing confinement ratio not only promotes the deformations of both constituent droplets, but also makes them more synchronous with applied shear. It is also found that the maximum deformation parameters of both droplets increase linearly with $\mathrm{Ca}$ up to $\mathrm{Ca}=0.35$ but deviate from the linearity at higher $\mathrm{Ca}$, where multipeaked oscillations are observed for the deformation of the inner droplet, which can be due to the extensional flow resulting from the rapid contraction of the outer droplet. We then analyze the breakup behavior of compound droplet in the oscillatory shear flow for varying confinement ratios, and compare the findings with those in simple shear flow. The critical capillary number for droplet breakup exhibits a non-monotonic behavior with the confinement ratio in both shear flows, but its value is always higher in oscillatory shear flow than in simple shear flow. As the confinement ratio increases, in the case of oscillatory shear flow, the droplet undergoes a transition from inner ternary breakup to inner binary breakup, distinct from the one observed in the case of simple shear flow. Finally, increasing oscillatory period is found to not only decrease the critical capillary number but also change the mode of droplet breakup.
\end{abstract}

(c) 2020 Elsevier Ltd. All rights reserved.

\section{Introduction}

A compound droplet, also known as a double emulsion, is a droplet of liquid containing one or more smaller droplets (inner droplets) within the main droplet in ring shape (outer droplet). The compound droplets have drawn much attention over the past decade due to their remarkable capacity and great potential in encapsulation and controlled release of active ingredients, separation and extraction processes, microreactors for chemical reactions, and these functions make them play an important role in applications such as materials processing, drug delivery, food processing, phar-

\footnotetext{
* Corresponding author.

E-mail address: haihu.liu@mail.xjtu.edu.cn (H. Liu).
}

maceuticals, personal care products and cosmetics (Utada et al., 2005; Shah et al., 2008; Lee and Weitz, 2008; Kim et al., 2011; Chen et al., 2011b; Wang et al., 2013). Compound droplets are also relevant in natural phenomena (Balla et al., 2020; Deka et al., 2019; Aston, 1972). The dynamic behaviors such as deformation and breakup are commonly experienced by compound droplets in these applications, which strongly influence the sizes and morphologies of compound droplets and in turn their properties and functions. Therefore, exploring the deformation and breakup of compound droplets is of great significance, which not only helps to understand the underlying physics, but also provides useful information that can be translated to industrial applications.

Compound droplets have been extensively studied from theoretical, experimental and numerical points of view. Some of the 
studies focused on the deformation and breakup of compound droplets that flow through confined geometries. For example, the dynamics of a compound droplet moving inside a circular tube was theoretically analyzed (Song et al., 2010) and numerically simulated (Borthakur et al., 2018; Che et al., 2018). Numerical studies have also been carried out to examine the morphological evolution of a single-core compound droplet and multiple emulsions with complex structures as they migrate through a gradually contracted tube where the flow is inhomogeneous (Zhou et al., 2008; Tao et al., 2013; Wang et al., 2014). Breakup of double emulsions when flowing through an orifice of a tapered nozzle was experimentally studied (Chen et al., 2011a; Li et al., 2011). On the other hand, a number of works investigated the behavior of compound droplets in an external flow, which include the deformation and breakup of a compound droplet in an extensional flow (Stone and Leal, 1990; Qu and Wang, 2012; Wang et al., 2013), and the motion of a compound multiphase droplet subject to a uniform stream (Sadhal and Oguz, 1985; Oguz and Sadhal, 1987). In addition, a few numerical and experimental works were dedicated to the deformation and breakup of a compound droplet in simple shear flow. Hua et al. (2014) numerically investigated the deformation dynamics of a compound droplet using two- and three-dimensional immersed boundary methods. They studied the effect of radius ratio, interfacial tension ratio and wall confinement, and compared the results with those obtained in the case of a single-phase droplet. Smith et al. (2004) used a level set method to study the deformation and breakup of a compound droplet in simple shear flow, where a phase diagram was established to describe the morphologies produced over a range of capillary numbers and core interfacial tensions. Chen et al. (2013) conducted numerical studies on the detailed rheological behaviors of double emulsion droplets and found four types of breakup modes via three mechanisms. Later, they combined experimental observations and numerical simulations to explore how the presence of inner droplet influences the deformation of a compound droplet in simple shear flow (Chen et al., 2015a), and identified two types of transient deformation topologies (Chen et al., 2015b). Recently, Vu et al. (2018, 2019) used a two-dimensional front-tracking method to study both deformation and breakup of single-core and multi-core compound droplets in simple shear flow. They considered the transition from non-breakup to breakup and revealed various patterns of deformation and breakup for a two-core compound droplet. Tiribocchi et al. (2020) used a lattice Boltzmann and finite difference hybrid method to investigate the steady states of a non-coalescing multi-core compound droplet under simple shear flow, and found that for a two-core compound droplet, the inner droplets periodically rotate around the mass center of the outer one, exhibiting an oscillatory steady state. Zhu and Gallaire (2017) numerically studied the dynamics of a particleencapsulated droplet under simple shear and discovered two new bifurcation scenarios that the particle executes spanwise migration and/or in-plane orbiting motion. However, the imposed external flows in the above studies are all limited to the time-independent cases. Particularly, little information is available in literature on the dynamics of a compound droplet when subjected to oscillatory shear flow, which is commonly used to measure the viscoelastic properties of complex fluids and serves as a building block of emulsion models (Cavallo et al., 2003; Farutin and Misbah, 2012).

Different from the case of compound droplets, single-phase droplets undergoing oscillatory shear flow have been explored since the pioneering theoretical work by Oldroyd (1953). Wannaborworn et al. (2002) performed experiments combined with numerical simulations to study the deformation and breakup of droplets under oscillatory shear for the viscosity ratio of unity over a range of strain amplitudes and frequencies. Cavallo et al. (2003) and Guido et al. (2004) experimentally investigated the deformation of an isolated Newtonian droplet subjected to small- and large-amplitude oscillatory shear flows respectively, and compared their findings with those from the theoretical predictions. Janpaen et al. (2009) carried out an experimental investigation of droplet deformation and breakup in oscillatory shear flows, where the immiscible components they used are Newtonian fluids as well as the Boger fluids. In addition, there are a few studies focusing on the deformation dynamics of spherical and non-spherical capsules in oscillating shear flow (Zhao and Bagchi, 2011; Matsunaga et al., 2015; Zhu et al., 2015). However, owing to the existence of inner droplet, it was found that compound droplets, compared with single-phase droplets, exhibit different topological structures and more complex behaviors (Smith et al., 2004; Gao and Feng, 2011; Hua et al., 2014; Luo et al., 2015; Chen et al., 2015b; Borthakur et al., 2018). Therefore, it is necessary to put effort into the deformation and breakup of compound droplets in oscillatory shear flow, which still remains unexplored thus far.

In the present work, we for the first time carry out a systematic numerical investigation on the deformation and breakup of a compound droplet in three-dimensional oscillatory shear flow. A lattice Boltzmann (LB) colour-gradient model recently developed by Yu et al. (2019) is adopted for the numerical investigation and its capability is verified by comparison with the available literature data. To complement the existing studies, we will explore not only the oscillatory deformation of compound droplet for varying oscillatory period, viscosity ratios, wall confinement and capillary number but also the critical conditions and modes of droplet breakup for varying wall confinement, in which the results are compared with those observed in simple shear flow. The rest of the paper is organized as follows. The problem statement and numerical method with the model validation and grid independence test are discussed in the next Section, and the results of droplet deformation in the oscillatory and breakup regimes are presented in Sections 3 and 4, respectively. The paper closes with a summary of the main results in Section 5.

\section{Problem statement and numerical method}

\subsection{Problem statement}

Consider a compound droplet immersed in a time-dependent oscillatory shear flow and undergoing deformation and breakup. The problem setup is depicted in Fig. 1. A Cartesian threedimensional coordinate system $(x, y, z)$ is used to formulate the problem, wherein $x, y$ and $z$ are the streamwise, spanwise and vertical directions, respectively. Initially, a spherical compound droplet, consisting of an outer droplet with radius $R_{0}$ and an inner droplet with radius $R_{i}$, is placed in the center of two infinite walls, both parallel to the $x-y$ plane and separated by a distance of $L_{z}$. The top and bottom walls move with equal sinusoidally oscillating speed but in opposite directions to impose the oscillatory shear flow. The oscillatory period is denoted as $T_{o s c}$, so the speed of the top wall is given by

$u_{w}(t)=U \sin \left(2 \pi t / T_{o s c}\right)$,

and further the shear rate $\dot{\gamma}$ can be calculated as

$\dot{\gamma}(t)=\dot{\gamma}_{\max } \sin \left(2 \pi t / T_{\text {osc }}\right)$,

where $\dot{\gamma}_{\max }=2 U / L_{z}$ is the shear rate amplitude. For the convenience of later discussion, the matrix fluid, outer droplet fluid and the inner droplet fluid are denoted as the fluid 1, fluid 2, and the fluid 3, respectively. They are all considered as immiscible, incompressible, Newtonian fluids with the dynamic viscosities $\mu_{k}(k=1,2,3)$. The immiscible interfaces are described by the interfacial tensions $\sigma_{k l}(k \neq l)$, and all three fluids are assumed to have equal densities $\rho_{k}$ so that buoyancy effects can be negligible. 


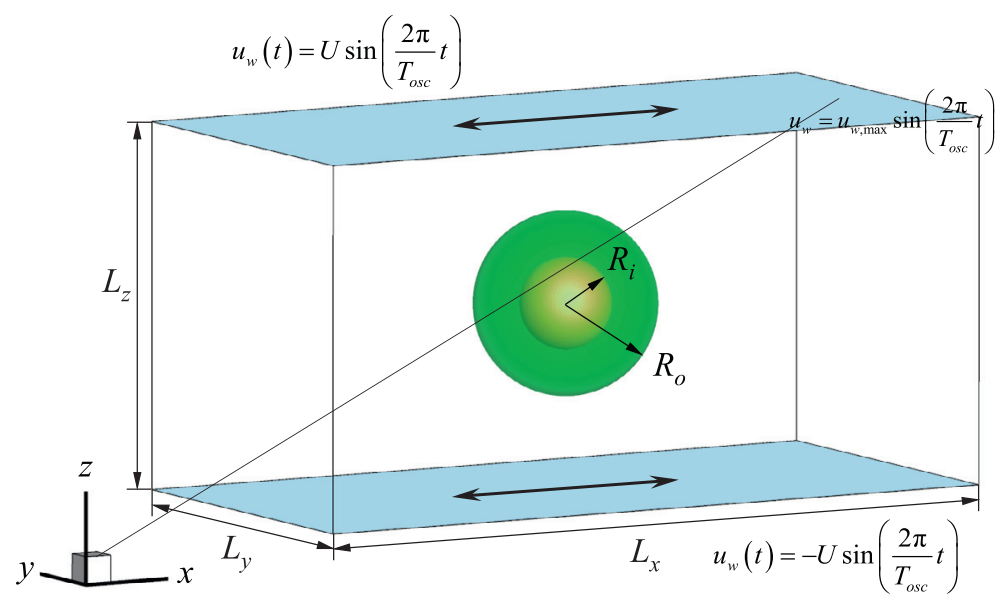

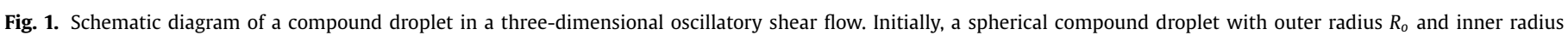
$R_{i}$ is placed in the center of the computational domain, which has a size of $L_{x} \times L_{y} \times L_{z}$.

This problem is simulated by a recently developed LB colorgradient model (discussed in the next subsection). The numerical simulations are conducted in a three-dimensional computational domain of size $L_{x} \times L_{y} \times L_{z}$, as shown in Fig. 1. The initial velocity $\boldsymbol{u}$ is set to zero everywhere in the computational domain. The boundary conditions are imposed as follows. In the $x$ and $y$ directions, the periodic boundary conditions are used; while on the top and bottom walls in the $z$ direction, the velocity boundary conditions (Zou and He, 1997) are imposed with velocity vector, $\boldsymbol{u}=\left(U \sin \left(2 \pi t / T_{o s c}\right), 0,0\right)$ and $\left(-U \sin \left(2 \pi t / T_{o s c}\right), 0,0\right)$, respectively.

To derive the dimensionless numbers associated with this problem, we use the initial radius of the outer droplet $\left(R_{0}\right)$, the maximum velocity of the droplet top $\left(\dot{\gamma}_{\max } R_{0}\right)$ and the oscillatory period $\left(T_{\text {osc }}\right)$ as the characteristic scales for length, velocity and time, respectively. The dimensionless parameters that govern the dynamics of the compound droplet can be defined as follows: the Reynolds number $R e=\rho_{1} \dot{\gamma}_{\max } R_{o}^{2} / \mu_{1}$, the capillary number $C a=\mu_{1} \dot{\gamma}_{\max } R_{0} / \sigma_{12}$, the radius ratio $R_{i} / R_{0}$, the wall confinement ratio $2 R_{o} / L_{z}$, the viscosity ratios $\lambda_{21}=\mu_{2} / \mu_{1}$ and $\lambda_{31}=\mu_{3} / \mu_{1}$, and the interfacial tension ratio $\lambda_{\text {int }}=\sigma_{12}: \sigma_{13}: \sigma_{23}$. Two parameters, namely deformation parameter $D$ and rotation angle $\theta$, are used to quantitatively characterize the deformation of the compound droplet in the $x-z$ mid-plane, since the side-view observation is the most revealing in terms of the mechanism involved (Wannaborworn et al., 2002). Following Taylor (1934), the deformation parameters of the outer and inner droplets from the side view are defined as $D_{o / i}=\left(L_{o / i}-B_{o / i}\right) /\left(L_{o / i}+B_{o / i}\right)$, where $L_{o / i}$ and $B_{o / i}$ represent the length of the major and minor axes of the outer/inner droplet, respectively. The angle of rotation, $\theta_{o / i}$, is defined as the angle between the velocity gradient axis and the droplet principle axis of extension. The sign of $\theta_{o / i}$ is used to distinguish the orientation of the droplet, so $\theta_{o / i}$ changes sign every half an oscillatory cycle.

\subsection{Numerical method}

A LB color-gradient model, recently developed by Yu et al. (2019) for ternary fluid flows, is used for our simulations. This model not only inherits many merits of its two-phase counterpart (Halliday et al., 2006, 2007), such as low spurious currents, strict mass conservation for each fluid, and good numerical stability for a broad range of viscosity ratios, but also is applicable to the fluids with a full range of interfacial tensions (no matter whether three interfacial tensions satisfy a Neumann triangle or not). Recently, it was extended to the simulation of three-phase flows with moving contact lines (Yu et al., 2019). Following Yu et al. (2019), three distribution functions $\left(f_{\alpha, 1}, f_{\alpha, 2}\right.$ and $\left.f_{\alpha, 3}\right)$ are introduced to represent the red (inner droplet), green (outer droplet) and blue (matrix fluid) fluids, respectively. The total distribution function is defined as $f_{\alpha}=\sum_{k} f_{\alpha, k}$, where the subscript $\alpha$ is the lattice velocity direction and ranges from 0 to 18 for the three-dimensional 19-velocity (D3Q19) lattice model used in this study. More information about the D3Q19 model, such as the lattice velocities $\boldsymbol{e}_{\alpha}$ and weight coefficients $w_{\alpha}$, can be found in Succi (2001); Guo and Shu (2013); Krüger et al. (2017).

The present color-gradient model consists of three steps, i.e. collision, recoloring and propagation. First, the total distribution function undergoes a collision step as

$f_{\alpha}^{\dagger}(\boldsymbol{x}, t)=f_{\alpha}(\boldsymbol{x}, t)-\frac{1}{\tau_{f}}\left[f_{\alpha}(\boldsymbol{x}, t)-f_{\alpha}^{e q}(\boldsymbol{x}, t)\right]+\Phi_{\alpha}(\boldsymbol{x}, t)$,

where $f_{\alpha}(\boldsymbol{x}, t)$ is the total distribution function in the $\alpha$ th velocity direction at the position $\boldsymbol{x}$ and time $t, f_{\alpha}^{\dagger}$ and $f_{\alpha}^{e q}$ are the post-collision and equilibrium distribution functions respectively, $\tau_{f}$ is the dimensionless relaxation time, and $\Phi_{\alpha}$ is the perturbation term.

The equilibrium distribution function is defined as a polynomial form with respect to the local fluid velocity vector $\boldsymbol{u}$ and is given by

$f_{\alpha}^{e q}(\boldsymbol{x}, t)=w_{\alpha} \rho\left[1+\frac{\boldsymbol{e}_{\alpha} \cdot \boldsymbol{u}}{c_{s}^{2}}+\frac{\left(\boldsymbol{e}_{\alpha} \cdot \boldsymbol{u}\right)^{2}}{2 c_{s}^{4}}-\frac{\boldsymbol{u}^{2}}{2 c_{s}^{2}}\right]$,

where $\rho=\sum_{k} \rho_{k}$ is the total density, and $c_{s}=1 / \sqrt{3}$ is the speed of sound.

The perturbation term contributes to the mixed interfacial regions and generates the interfacial tensions between different fluids. The perturbation term is given by (Guo et al., 2002; Halliday et al., 2006)

$\Phi_{\alpha}(\boldsymbol{x}, t)=w_{\alpha}\left(1-\frac{1}{2 \tau_{f}}\right)\left[\frac{\boldsymbol{e}_{\alpha}-\boldsymbol{u}}{c_{s}^{2}}+\frac{\left(\boldsymbol{e}_{\alpha} \cdot \boldsymbol{u}\right) \boldsymbol{e}_{\alpha}}{c_{s}^{4}}\right] \cdot \boldsymbol{F}_{s}(\boldsymbol{x}, t) \delta_{t}$,

where $\delta_{t}$ is the time step and the local velocity is defined by $\rho \boldsymbol{u}(\boldsymbol{x}, t)=\sum_{\alpha} f_{\alpha}(\boldsymbol{x}, t) \boldsymbol{e}_{\alpha}+\frac{1}{2} \boldsymbol{F}_{s}(\boldsymbol{x}, t) \delta_{t}$. The interfacial force $\boldsymbol{F}_{s}$ can be derived from the perturbation operator proposed by Leclaire et al. (2013), which reads as (Yu et al., 2019)

$\boldsymbol{F}_{S}=\sum_{k} \sum_{l, l \neq k} \nabla \cdot\left[\frac{\sigma_{k l} C_{k l}}{2}\left|\boldsymbol{G}_{k l}\right|\left(\boldsymbol{I}-\boldsymbol{n}_{k l} \boldsymbol{n}_{k l}\right)\right]$ 


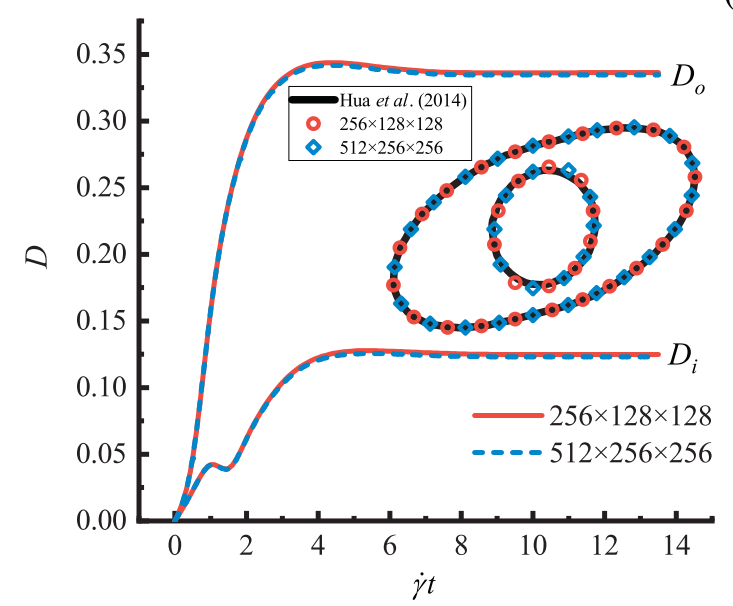

(a)

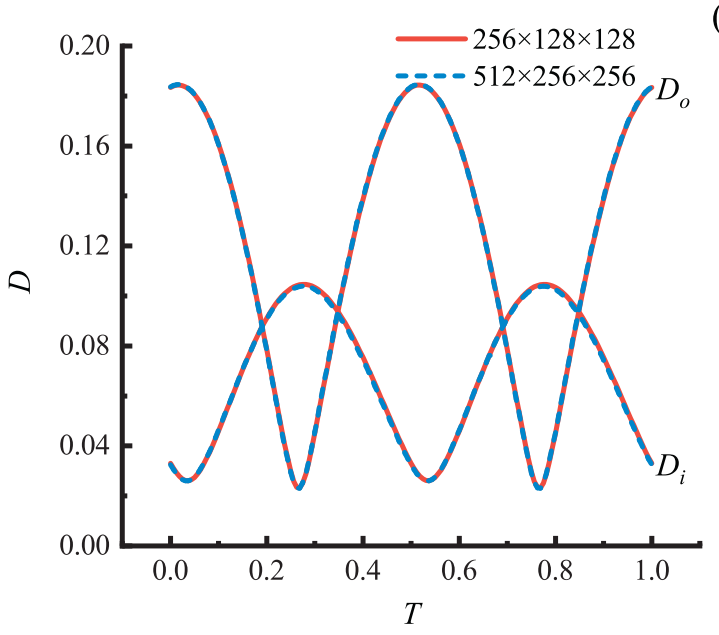

(b)

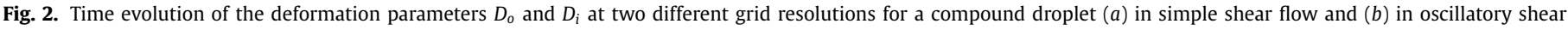

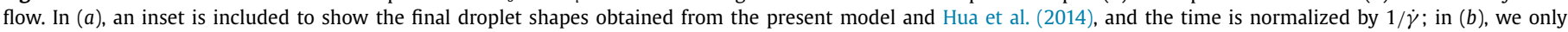
show the results in one oscillatory cycle when both inner and outer droplets reach the steady-state oscillations, and the dimensionless time is defined as $T=t / T_{\text {osc }}$.

where $\boldsymbol{G}_{k l}=\frac{\rho_{l}}{\rho} \nabla \frac{\rho_{k}}{\rho}-\frac{\rho_{k}}{\rho} \nabla \frac{\rho_{l}}{\rho}$ is the color gradient, $\boldsymbol{n}_{k l}=\boldsymbol{G}_{k l} /\left|\boldsymbol{G}_{k l}\right|$ is the unit normal vector of the $k-l$ interface, $C_{k l}$ is a concentration factor controlling the activation of the interfacial tension at the $k-$ $l$ interface, and $\boldsymbol{I}$ is the second-order identity tensor.

Using the Chapman-Enskog expansion, it can be shown that Eqs. (3)-(5) exactly recover the Navier-Stokes equations for ternary fluid flow, where the pressure and the dynamic viscosity of the fluid mixture are defined by $p=\rho c_{s}^{2}$ and $\mu=\left(\tau_{f}-0.5\right) \rho c_{s}^{2} \delta_{t}$. To allow for unequal viscosities of the three fluids, we determine the viscosity $\mu$ by a harmonic mean,

$\frac{\rho}{\mu}=\sum_{k} \frac{\rho_{k}}{\mu_{k}}$.

Then, a recoloring step is applied to minimize the mixing of different fluids (Gunstensen et al., 1991; Latva-Kokko and Rothman, 2005). Following Spencer et al. (2010), the recolored distribution function of the fluid $k$ is

$f_{\alpha, k}^{\ddagger}(\boldsymbol{x}, t)=\frac{\rho_{k}}{\rho} f_{\alpha}^{\dagger}(\boldsymbol{x}, t)+\sum_{l, l \neq k} \beta_{k l} w_{\alpha} \frac{\rho_{k} \rho_{l}}{\rho} \boldsymbol{n}_{k l} \cdot \boldsymbol{e}_{\alpha}$,

where $f_{\alpha, k}^{\ddagger}$ is the recolored distribution function of the fluid $k ; \beta_{k l}$ is a segregation parameter related to the thickness of the $k-l$ interface, and it is defined as (Yu et al., 2019)

$\beta_{k l}=\beta_{0}+\beta_{0} \min \left(\frac{35 \rho_{1} \rho_{2} \rho_{3}}{\rho^{3}}, 1\right) g\left(X_{k l}\right)$.

Here,

$g\left(X_{k l}\right)= \begin{cases}1, & X_{k l}<1 \\ 1-\sin \left[\arccos \left(X_{k l}\right)\right], & -1 \leqslant X_{k l}<0 \\ \sin \left[\arccos \left(X_{k l}\right)\right]-1, & 0 \leqslant X_{k l} \leqslant 1 \\ -1, & 1<X_{k l},\end{cases}$

where $X_{k l}=\left(\sigma_{m k}^{2}+\sigma_{m l}^{2}-\sigma_{k l}^{2}\right) /\left(2 \sigma_{m k} \sigma_{m l}\right)$ is a parameter that determines whether the Neumann triangle exists; $\beta_{0}$ is the reference segregation parameter, and is fixed at 0.7 to be consistent with the segregation parameter in two-phase color-gradient models (Halliday et al., 2007; Liu et al., 2012).

After the recoloring step, in the propagation step, the three distribution functions $\left(f_{\alpha, 1}, f_{\alpha, 2}\right.$ and $\left.f_{\alpha, 3}\right)$ propagate to the neighboring lattice sites, which is done as

$f_{\alpha, k}\left(\boldsymbol{x}+\boldsymbol{e}_{i} \delta_{t}, t+\delta_{t}\right)=f_{\alpha, k}^{\ddagger}(\boldsymbol{x}, t), \quad k=\{1,2,3\}$,

with the post-propagation distribution functions used to compute the densities of the constituent fluids by $\rho_{k}=\sum_{\alpha} f_{\alpha, k}$.

\subsection{Validation and grid independence test}

In our previous work, the present model has been validated by several static tests, including the Young-Laplace test for a compound droplet and the spreading of a droplet between two stratified fluids, and it was demonstrated to accurately reproduce the stability diagram of double droplets over a wide range of interfacial tensions (Yu et al., 2019). Later, it was also validated against the analytical solutions by dynamic problems, e.g. dynamic capillary filling of ternary fluids (Yu et al., 2019). Here, we further validate the model by simulation of a dynamic problem and perform grid independent test so that a proper grid resolution can be determined.

First, we simulate the deformation of a compound droplet in a simple shear flow where the shear rate is a constant and is given by $\dot{\gamma}=2 U / L_{z}$, and compare the simulation results with those obtained by Hua et al. (2014). The size of the computational domain is $L_{x} \times L_{y} \times L_{z}=8 R_{0} \times 4 R_{0} \times 4 R_{0}$, and the radius ratio $R_{i} / R_{0}$ and wall confinement ratio $2 R_{o} / L_{z}$ are both set to $\frac{1}{2}$. The simulations are performed with $R e=\rho_{1} \dot{\gamma} R_{0}^{2} / \mu_{1}=1, C a=\mu_{1} \dot{\gamma} R_{0} / \sigma_{12}=0.25$, $\lambda_{21}=\lambda_{31}=1$ and $\lambda_{\text {int }}=1: 1: 1$ for the grid resolutions of $R_{0}=32$ and $R_{0}=64$, and the simulation results are shown in Fig. 2(a). It is seen that both grid resolutions produce almost the same results in terms of the temporal evolution of $D_{o / i}$ and the final shapes of inner and outer droplets. Also, the final shapes of inner and outer droplets agree well with the results obtained by Hua et al. (2014), which indicates that the present model is capable of simulating dynamic problems with satisfactory accuracy.

We then turn to the oscillatory shear flow and carry out a grid independence test. All the simulation parameters are the same as those in the validation test except that the oscillatory shear flow with $T_{o s c}=L_{X} / U$ is imposed instead of the simple shear flow. In the simulations, we record the temporal evolutions of the deformation parameters, $D_{o / i}$ and the angles of rotation, $\theta_{o / i}$ of the outer and inner droplets. When the maximum relative difference of $D_{o / i}$ and $\theta_{o / i}$ at the corresponding instants of two adjacent oscillatory cycles is less than $5 \times 10^{-6}$, we assume that the system reaches the steady state and the relevant data are extracted for further analysis. The deformation parameters $D_{o / i}$ in one steady-state oscillatory cycle obtained from the grid resolutions of $R_{0}=32$ and $R_{0}=64$ are shown in Fig. 2(b). It is clear that the two groups of curves nearly overlap with each other. This indicates that the grid resolution of $R_{0}=32$ is fine enough to produce grid-independent results 


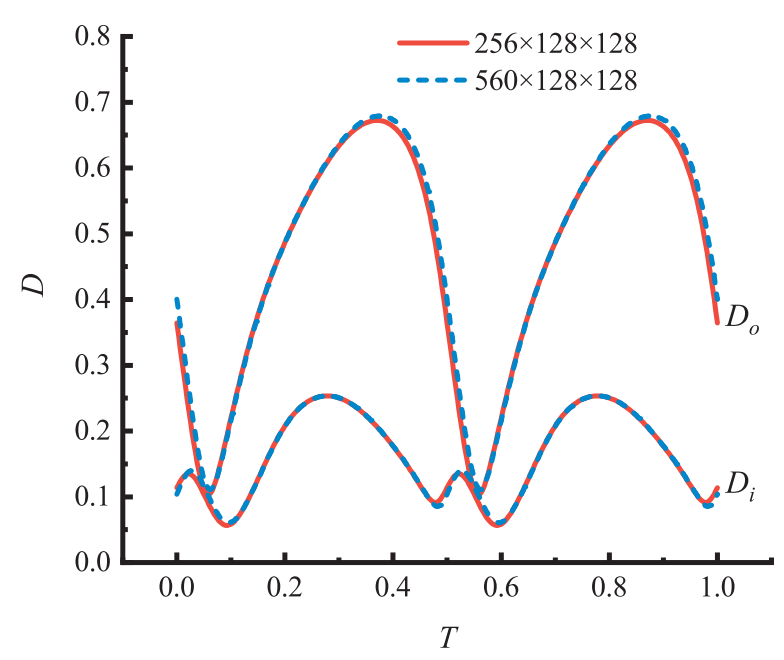

Fig. 3. Time evolution of deformation parameters $D_{0}$ and $D_{i}$ in one steady-state oscillatory cycle for the grid systems of $8 R_{0} \times 4 R_{0} \times 4 R_{0}$ and $16 R_{0} \times 4 R_{0} \times 4 R_{0}$. The simulations are run with $R_{i} / R_{0}=2 R_{o} / L_{z}=\frac{1}{2}, \lambda_{21}=\lambda_{31}=1, R e=1, C a=0.45$ and $T_{\text {osc }}=10 L_{x} / U$. The dimensionless time $T$ is defined as $T=t / T_{\text {osc }}$.

under the oscillatory shear flow, so this grid has been adopted to perform the rest of the simulations. Besides, it is found that the droplet may touch itself via the periodic boundary conditions in the breakup cases when the length of the computational domain $L_{x}$ is taken as $8 R_{0}$. To avoid this, we lengthen the computational domain to $L_{X}=16 R_{O}$ while studying the droplet breakup. It should be noted that the value of $L_{x}$ does not affect the droplet behavior as long as the droplet front keeps a distance of at least $R_{0}$ away from the periodic boundaries. This can be demonstrated by the simulation results plotted in Fig. 3, where we observe that increasing $L_{x}$ from $8 R_{0}$ to $16 R_{0}$ leads to slight change of the deformation parameters $D_{o / i}$ in one steady-state oscillatory cycle.

\section{Oscillatory deformation of a compound droplet}

\subsection{Effect of oscillatory period}

First, we investigate the effect of the oscillatory period $T_{\text {osc }}$ on the deformation of a compound droplet with $R_{i} / R_{0}=2 R_{0} / L_{z}=\frac{1}{2}$, $R e=1, C a=0.25$ and viscosity ratios $\lambda_{21}=\lambda_{31}=1$. The oscilla- tory period is defined as $T_{o s c}=n L_{x} / U$, and different values of $T_{\text {osc }}$ are obtained by varying the parameter $n$ from 0.1 to 20. Following Wannaborworn et al. (2002), the characteristic relaxation time of the compound droplet is defined as $\tau_{\text {rel }}=\mu_{1} R_{o} / \sigma_{12}$. Then we can calculate the time scale ratio $\tau_{r}$ of the droplet relaxation time to the oscillation period of the external flow through $\tau_{r}=\tau_{\text {rel }} / T_{\text {osc }}$. It is found that for each oscillatory period, the compound droplet eventually reaches a steady-state oscillatory condition, where the droplet behavior no longer changes from one periodic time to the next. We record the time scale ratio, deformation parameters and rotation angles of both inner and outer droplets in the steadystate oscillatory condition, and the results are illustrated in Fig. 4, in which the steady-state results from the corresponding simple shear flow, where the top and bottom walls move with velocities $U$ and $-U$ respectively, are also shown for comparison. In Fig. 4(a), one can see that with the increase of oscillatory period, the maximum deformation parameters $D_{o \text {, max }}$ and $D_{i, \max }$ first increase rapidly, then undergo slow growth and finally reach certain steady values, which exactly correspond to the steady-state deformation parameters $D_{o \text {,max }}$ and $D_{i, \max }$ in the corresponding simple shear flow. This behavior can be explained through the time scale ratio $\tau_{r}$, which is plotted as a function of $n$ in Fig. 4(a). A small time scale ratio $\tau_{r}$ (corresponding to a large oscillatory period) means the droplet will relax very quickly compared to the time scale for deformation. Thus, at any time, the compound droplet gets enough time to approach its equilibrium shape for a particular shear rate and, in turn, the compound droplet undergoes a greater degree of deformation $\left(D_{i / o, \max }\right)$ in one oscillatory cycle. On the contrary, when the time scale ratio is high, the compound droplet cannot change its shape fast enough to reach the equilibrium shape, so the maximum deformation parameters, $D_{i / o \text {,max }}$ get smaller. We also note in Fig. $4(b)$ that $\theta_{\mathrm{m}, o}\left(\theta_{\mathrm{m}, i}\right)$ is the rotation angle when the outer (inner) droplet undergoes the maximum deformation. Similar to the maximum deformation parameters, the rotation angles $\theta_{\mathrm{m}, o}$ and $\theta_{\mathrm{m}, i}$ also exhibit a tendency to approach the steady-state rotation angles in simple shear flow, when the oscillatory period gets longer. These results also demonstrate that the simple shear is indeed a limiting case of the oscillatory shear when $T_{\text {osc }}$ becomes infinite.

Fig. 4(a) also shows the variation of the minimum deformation parameters $D_{o, \min }$ and $D_{i, \min }$ with the oscillatory period. It is observed that in some cases the values of $D_{o, \min }$ and $D_{i, \min }$ can be non-zero, suggesting that the outer or inner droplet cannot

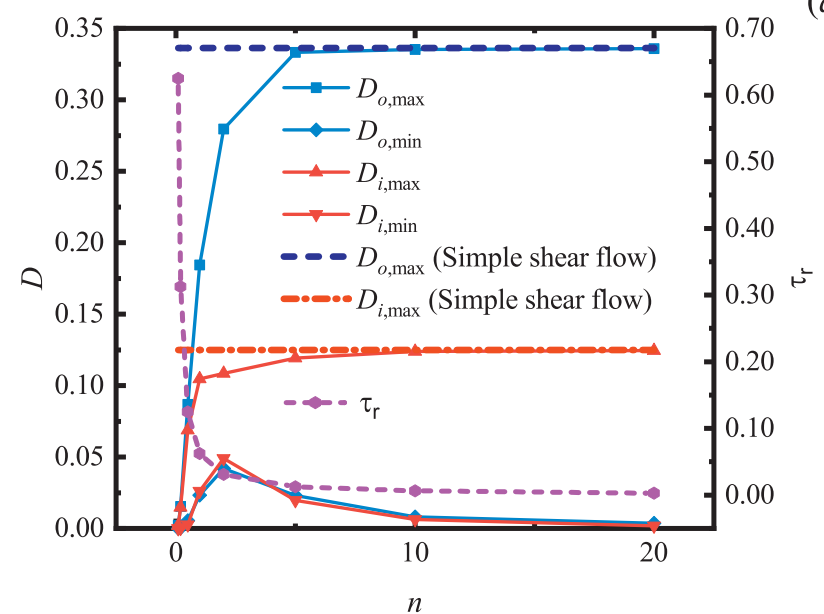

(a)

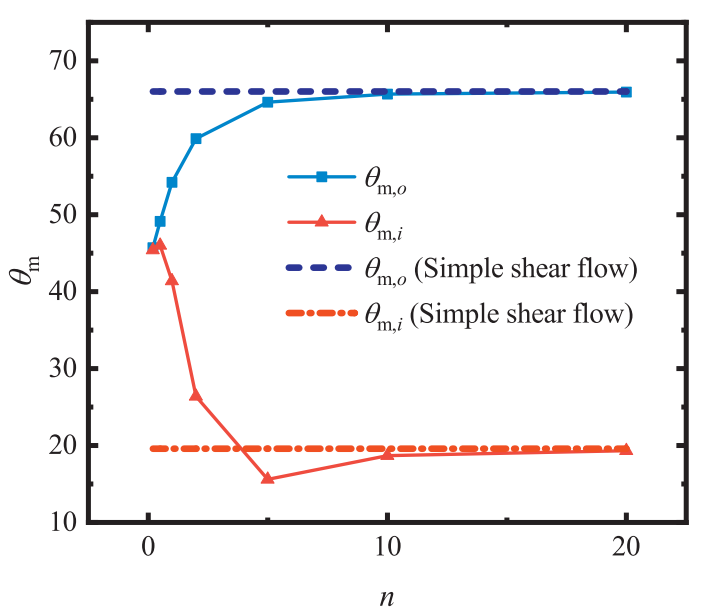

(b)

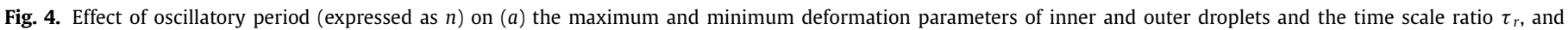

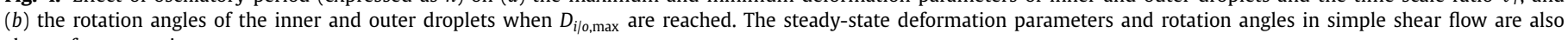
shown for comparison. 


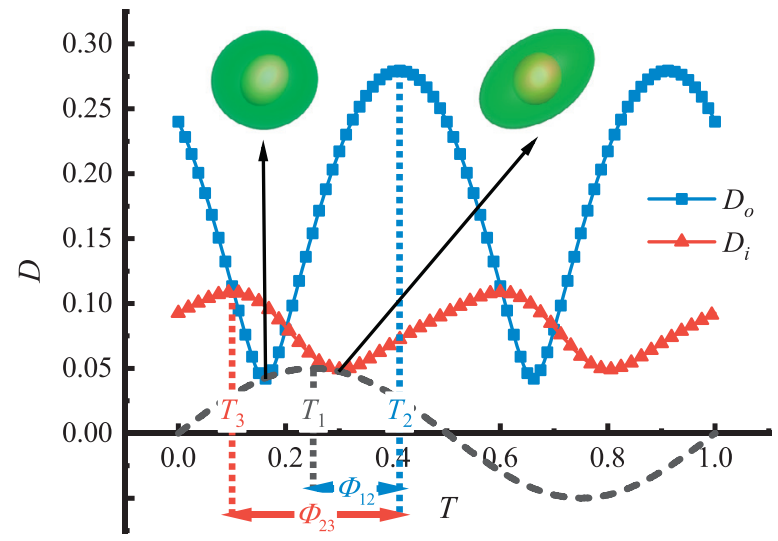

Fig. 5. Instantaneous deformation parameters of outer droplet $\left(D_{o}\right)$ and inner droplet $\left(D_{i}\right)$ in one oscillatory cycle when reaching steady-state oscillation for $n=2$. Time is normalized by the oscillatory period. The shear rate $\dot{\gamma}$ is shown by the black dashed lines in arbitrary scale, and the definitions of $\Phi_{12}$ and $\Phi_{23}$ are also illustrated.

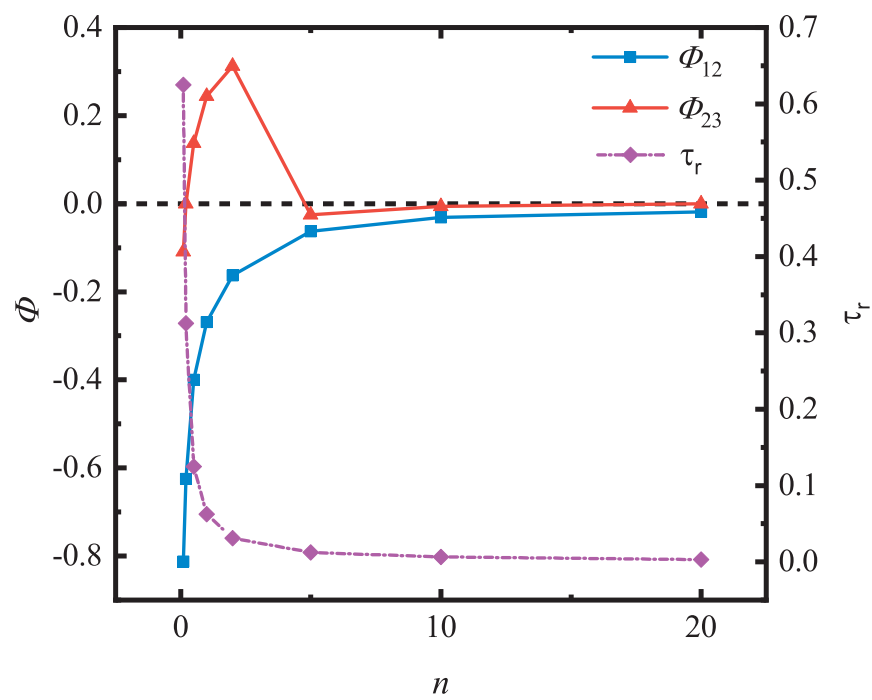

Fig. 6. Effect of oscillatory period (expressed as $n$ ) on the phase lags $\Phi_{12}$ and $\Phi_{23}$, which are normalized by the oscillatory period $T_{\text {osc }}$. The plot of $n$ versus $\tau_{r}$, directly taken from Fig. 4(a), is also depicted.

return to a truly spherical shape during the steady-state oscillation. A similar observation was reported by Wannaborworn et al. (2002) in the case of a single-phase droplet in an oscillatory shear flow. To clearly show the non-spherical minimum deformation, we plot the results of $n=2$ in Fig. 5, since the values of $D_{o, \text { min }}$ and $D_{i, \text { min }}$ are the largest at $n=2$ (see Fig. $4(a)$ ). Inspection of Fig. 5 reveals that the instantaneous deformation parameter $D_{o / i}$ varies between $D_{o / i, \min }$ and $D_{o / i \text { max }}$ with the shear flow. However, the minimum or maximum deformation parameters of the inner and outer droplets do not occur at the same time, and the maximum deformation of the compound droplet occurs after the shear flow reaches its maxima. This means that there exist the phase lag between the deformation responses of inner and outer droplets, and the phase lag between the deformation response of the compound droplet and applied shear, which are denoted as $\Phi_{23}$ and $\Phi_{12}$, respectively. By choosing three time points as reference in one oscillatory period, i.e. $T_{1}, T_{2}$ and $T_{3}$, which respectively correspond to $\dot{\gamma}=\dot{\gamma}_{\max }, D_{o}=D_{0, \max }$ and $\theta_{\mathrm{m}, o}>0$, and $D_{i}=D_{i, \max }$ and $\theta_{\mathrm{m}, i}>0$, we can compute $\Phi_{23}$ and $\Phi_{12}$ through $\Phi_{23}=T_{2}-T_{3}$ and $\Phi_{12}=T_{1}-T_{2}$ (see Fig. 5).
Fig. 6 shows the variations of $\Phi_{12}$ and $\Phi_{23}$ as a function of oscillatory period (expressed as $n$ ), in which the corresponding values of time scale ratio are plotted as well. For all values of $n$ considered, $\Phi_{12}$ is negative, indicating that the deformation response of the compound droplet always lags behind the applied shear. As the oscillatory period increases, $\Phi_{12}$ gets closer to zero. This is because the droplet responds quickly to the time-varying external flow for a smaller time scale ratio, which enhances the synchronization between the outer droplet and the applied shear. A similar result was also found by Zhao and Bagchi (2011) even though they considered a deformable capsule in oscillatory shear flow. However, unlike $\Phi_{12}, \Phi_{23}$ exhibits a non-monotonic variation with $n$ : first increasing, then decreasing, and finally slightly increasing again to zero, where the maximum of $\Phi_{23}$ occurs at $n=2$, with a value of 0.313 . Evidently, the variation of $\Phi_{23}$ with $n$ is identical to that of $D_{i, \min }$ (see Fig. $4(a)$ ) in trend, suggesting that the asynchronization of deformation response between the inner and outer droplets is responsible for the non-zero values of $D_{i, \min }$, as will be further explained below. In addition, we can see that when $n \geq 10$, the deformation of outer droplet follows closely the applied oscillatory shear $\left(\Phi_{12} \approx 0\right)$, and at the same time the deformation of inner droplet follows closely that of the outer droplet $\left(\Phi_{23} \approx 0\right)$. In other words, the maximum deformations occur shortly after the shear rate reaches its maximum value, and both droplets resume the spherical shape soon after the zero shear rate. With regard to the rotation, the outer droplet aligns well with the oscillatory shear flow, while the inner droplet exhibits relatively complex behavior, which will be shown below.

As an example, Fig. 7(a) shows the instantaneous deformation parameters $D_{0}$ and $D_{i}$ as well as the instantaneous rotation angle $\theta_{i}$ of the inner droplet in one steady oscillatory cycle for $n=10$. Considering the symmetry and periodicity of the dynamic responses, our attention is only paid to a quarter cycle, e.g. the time interval between $D_{0, \min }$ and $D_{o \text {,max. }}$. In this time interval, four instants are selected as representatives, i.e. $T=0.03$ (i), 0.09 (ii), 0.16 (iii) and 0.28 (iv), and the corresponding pressure distributions and velocity fields are depicted in Fig. $7(b)$. At $T=0.03$, the outer droplet returns to its minimum deformation shortly after the zero shear rate $(T=0)$, and at the same time the inner droplet deforms slightly and tilts to the right $\left(\theta_{i}>0\right)$. As the shear flow strengthens, the deformation and rotation angle of the outer droplet both increase, but the rotation angle of the inner droplet undergoes a rapid decrease from positive to negative values and reaches its minima at $T=0.09$ (ii). This rapid change of $\theta_{i}$ seems somewhat counterintuitive, which can be explained as follows. The deformation of the inner droplet $\left(D_{i}\right)$ is mainly induced by a closed vortical flow interior to the outer droplet (Chen et al., 2013; $\mathrm{Vu}$ et al., 2018), while its rotation angle $\theta_{i}$ is also affected by the pressure distribution inside the outer droplet. As the outer droplet becomes more and more stretched, the pressure inside it would be higher at the two farthest ends, which is to balance the interfacial tension resulting from the higher interface curvature. During the time $T=0.03$ to 0.09 , the pressure force is dominant over the vortical flow inside the outer droplet, which creates a torque to rotate the inner droplet in an anticlockwise direction, leading to a decrease in $\theta_{i}$ value. Upon further strengthening the shear flow, the inner droplet begins to rotate clockwise (iii), although the high pressure zones still exist inside the outer droplet. This is because (1) the high pressure zones move away from the inner droplet, weakening the influence of the pressure force, and (2) a stronger vortical flow is developed inside the outer droplet. It should be noted that $\theta_{i}$ keeps increasing with $D_{i}$ until they reach the maximum values simultaneously (iv). After undergoing the maximum deformation and rotation angle, the outer droplet retracts, and the inner droplet also becomes less deformed with a decrease in $\theta_{i}$. Thus, the compound droplet would return to the morphologies 


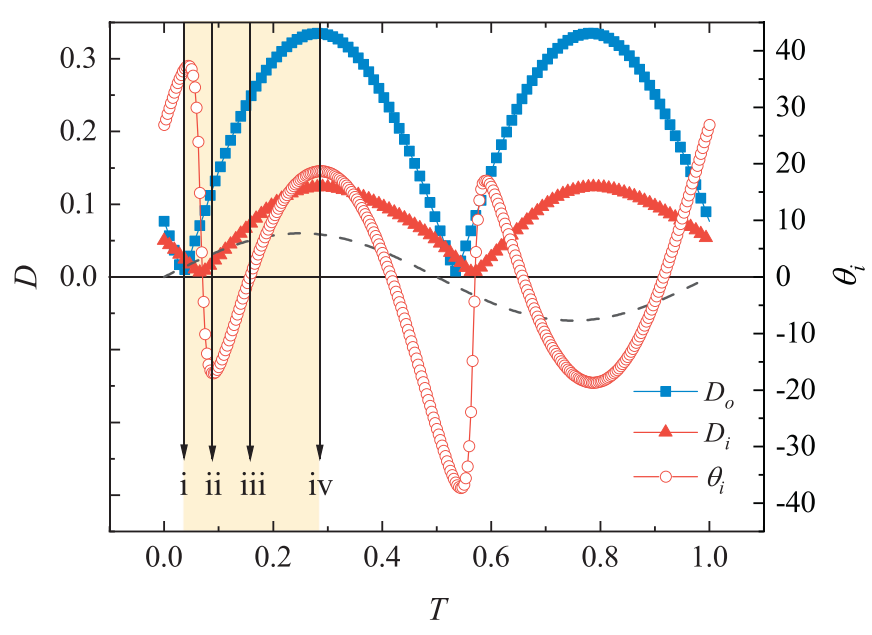

(a)

(b)
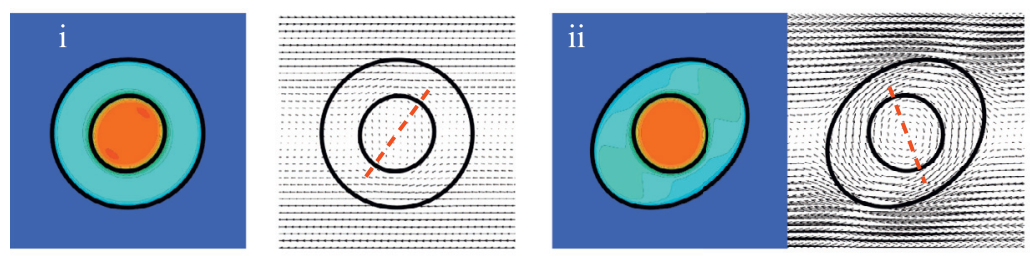

$P$
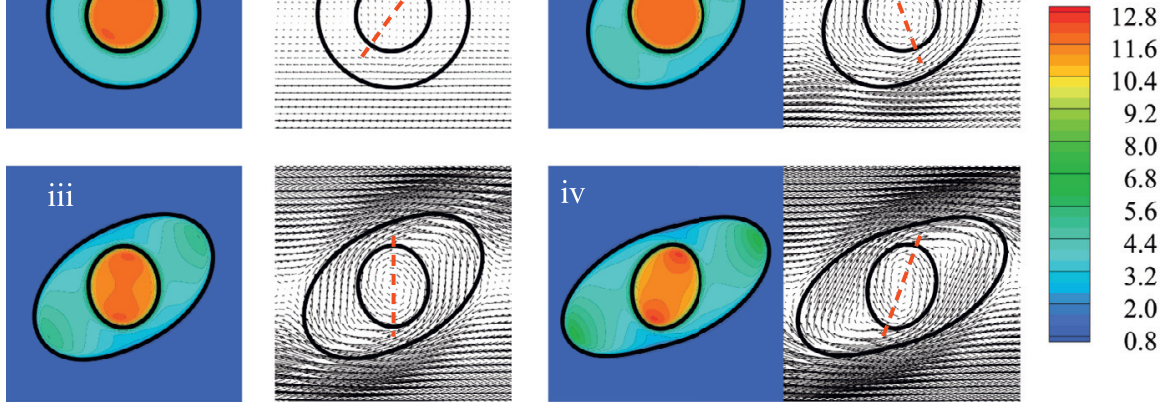

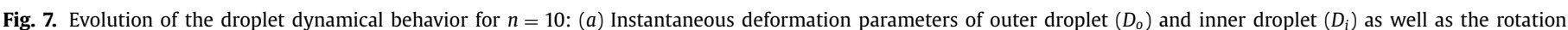

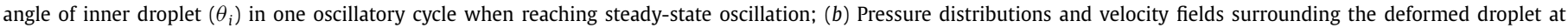

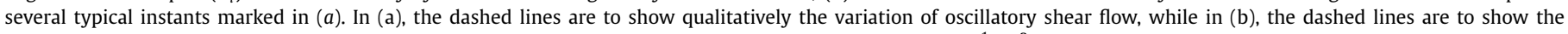
rotation angles of inner droplet. The time and pressure $P$ are normalized by the oscillatory period and and $\frac{1}{2} \rho U^{2}$, respectively.

shown in (iii) and (ii), and experience once more the sharp change of $\theta_{i}$ at around $T=0.5$, except that $\theta_{i}$ varies from negative to positive values at this time. According to the above results, we conclude that in one oscillatory cycle, the dynamic behaviour of the inner droplet is mainly controlled by the viscous shear force arising from the closed vortical flow interior to the outer droplet, but it is also affected by the pressure force especially during the reversal of shear flow.

As shown above, the asynchronization between the inner droplet and outer droplet is attributed to the competition of different forces acting on the inner droplet, which mainly include the viscous shear force arising from the closed vortical flow interior to the outer droplet and the pressure force exerted by the high pressure near two tips inside the outer droplet. When the pressure force becomes dominant, the inner droplet is forced to rotate in an opposite direction to the outer droplet before reaching a spherical shape, meaning that it cannot anyhow return to a truly spherical shape. As a result, the larger the pressure, the more asynchronous the inner and outer droplets (i.e. the bigger the phase lag $\Phi_{23}$ ) and, in turn, the bigger the minimum deformation $D_{i, \min }$ of the inner droplet, which can be clearly seen from the combination of Fig. 4(a) and 6.

\subsection{Effect of viscosity ratios}

Next, the effect of viscosity ratios on the behavior of compound droplet is investigated for the oscillatory period $T_{o s c}=L_{x} / U$ at
$R_{i} / R_{0}=2 R_{o} / L_{z}=\frac{1}{2}, R e=1$ and $C a=0.25$. Different values of $\lambda_{21}$ and $\lambda_{31}$, ranging from 0.1 to 2 , are achieved by varying $\mu_{2}$ and $\mu_{3}$ respectively while keeping $\mu_{1}$ fixed. We note that when the effect of $\lambda_{21}\left(\lambda_{31}\right)$ is investigated, the viscosity ratio $\lambda_{31}\left(\lambda_{21}\right)$ is fixed at 1 .

Fig. 8 shows the effect of the viscosity ratios $\lambda_{21}$ and $\lambda_{31}$ on the maximum deformation parameters $D_{o / i, \max }$ and the corresponding rotation angles $\theta_{\mathrm{m}, o / i}$. As $\lambda_{21}$ increases, which means the viscosity of the outer droplet increases, the outer droplet becomes less deformed but its rotation angle remains almost unchanged. The decrease in the deformation of the outer droplet is because the outer droplet is more like a solid as its viscosity increases. On the other hand, for the inner droplet, its maximum deformation and rotation angle both exhibit a substantial increase as $\lambda_{21}$ (i.e. $\mu_{2}$ ) increases, suggesting that the inner droplet becomes more deformed and tilts closer to the flow direction. This is attributed to the fact that increasing $\mu_{2}$ increases the viscous shear stresses exerted on the inner droplet, which stretch the droplet towards the flow direction. Compared with $\lambda_{21}, \lambda_{31}$ exhibits less influence on the deformations of both outer and inner droplets and the rotation angles, which can be seen from Fig. $8(a, b)$. This is understandable given that (1) increasing $\mu_{3}$ does not significantly change the driving force acting on either droplet, and (2) the inner droplet only occupies one eighth of the entire compound droplet while the outer droplet occupies seven eighths in volume.

The effect of both $\lambda_{21}$ and $\lambda_{31}$ on the phase lags is shown in Fig. 9. It is found that both $\Phi_{12}$ and $\Phi_{23}$, which are of op- 


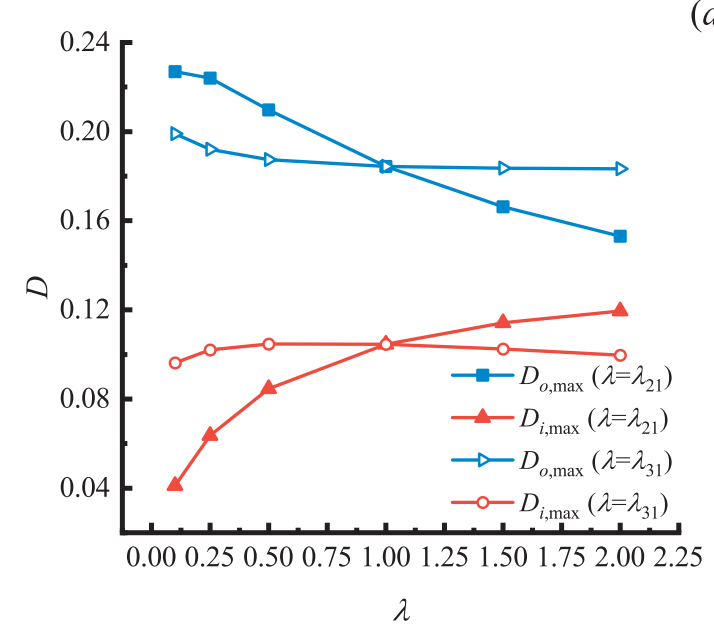

(a)

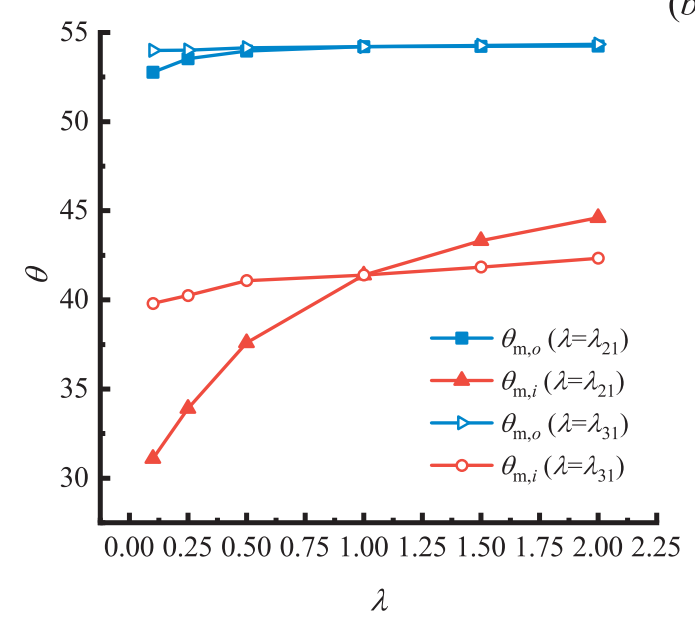

(b)

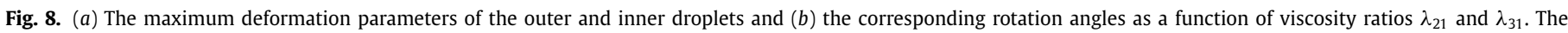
solid and hollow symbols represent the results obtained by varying $\lambda_{21}$ and $\lambda_{31}$, respectively.

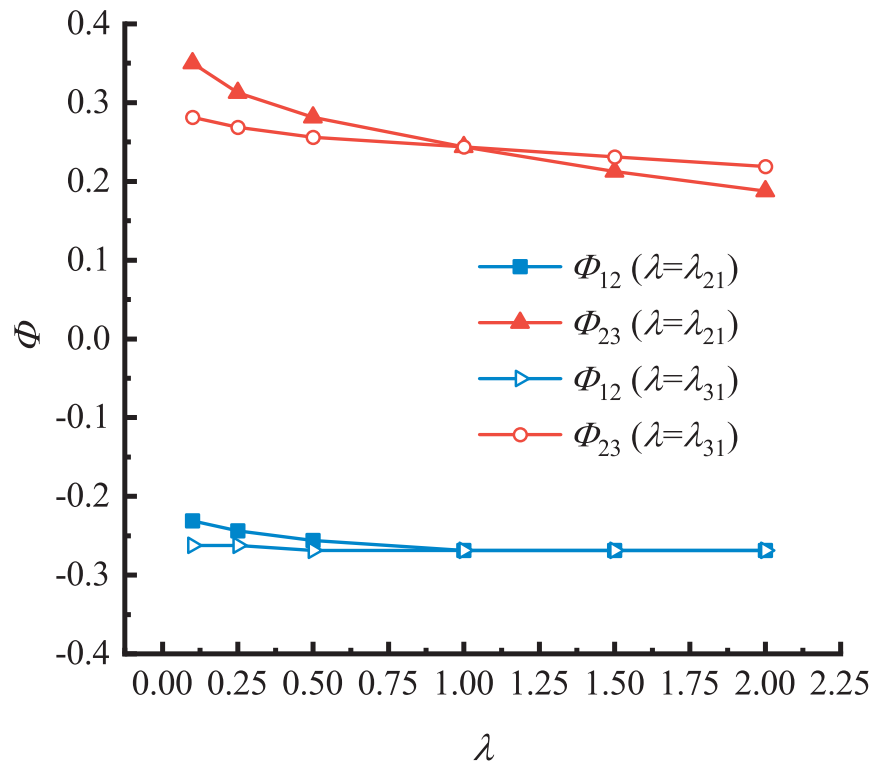

Fig. 9. Effect of the viscosity ratios $\lambda_{21}$ and $\lambda_{31}$ on $\Phi_{12}$ and $\Phi_{23}$. The solid and hollow symbols represent the results obtained by varying $\lambda_{21}$ and $\lambda_{31}$, respectively.

posite signs, decline to some extent with increasing viscosity ratios. In contrast to $\Phi_{23}, \Phi_{12}$ is less affected by the viscosity ratios, which is consistent with the variation of rotation angles shown in Fig. $8(b)$, where $\theta_{\mathrm{m}, o}$ is roughly a constant. This is likely because the orientation of the outer droplet is primarily dominated by the external oscillatory shear, whose amplitude and frequency remain unchanged when varying the viscosity ratios. In addition, it is noticed in Fig. 9 that $\Phi_{23}$ maintains relatively big positive values for various viscosity ratios, which suggests that the maximum deformation of the inner droplet occurs much earlier than that of the outer droplet, especially when $\lambda_{21}=0.1$. This is also demonstrated by the results in Fig. 10(a), where $\Phi_{23}$ is clearly illustrated. In order to better understand the asynchronization of deformation response between inner and outer droplets, we select three typical instants for analysis in a quarter cycle, i.e. (i)-(iii), and the corresponding pressure and velocity distributions around the deformed droplet are presented in Fig. 10(b). Under the action of the applied shear, the outer droplet rotates clockwise and resumes a nearly spherical shape at the time of (i), and at the same time the inner droplet inclines to the right. Then, the outer droplet undergoes a growing deformation, during which time two high pressure zones are formed near two tips inside the outer droplet. The high pressures squeeze the inner droplet and make it return to a spherical shape at the time of (ii). As the deformation of the outer droplet is further increased, the pressure force increases which drives the inner droplet to rotate anticlockwise, even though the vortical flow stretching the inner droplet towards the right also strengthens. Thus, it is seen in Fig. 10(b) that the inner droplet inclines to the left when the outer droplet reaches its maximum deformation at the time of (iii). Based on the above analysis, it is clear that the asynchronization between inner and outer droplets is caused by the dominant role of pressure force, while the vortical flow inside the outer droplet is responsible for decreasing the asynchronization. From this conclusion, one can expect that, if the vortical flow becomes stronger, like in the case of $\lambda_{21}=2$ where $D_{0, \max }$ is relatively small and the impact of high pressure zones is thus weakened, then the value of $\Phi_{23}$ would be smaller, consistent with the results shown previously in Fig. 9.

\subsection{Effect of wall confinement}

In this section, the effect of wall confinement on the deformation of compound droplet is studied for $R_{i} / R_{0}=\frac{1}{2}, R e=1, C a=$ $0.25, \lambda_{21}=\lambda_{31}=1$ and $T_{o s c}=10 L_{x} / U$. The confinement ratio $2 R_{o} / L_{z}$ is varied from 0.25 to 0.8 by adjusting the distance between two parallel walls.

Fig. 11(a) plots the maximum and minimum deformation parameters of the outer and inner droplets as a function of the confinement ratio. It is seen that as the confinement ratio increases, the maximum deformation parameters of inner and outer droplets both increase, but the deformation of outer droplet increases more significantly. This is because (1) the outer droplet is directly subjected to the applied shear flow, but the inner droplet is engulfed by the outer droplet which exerts a buffering effect between the inner droplet and the external shear flow; (2) compared to the outer droplet, the inner droplet has a lower confinement ratio, at which the effect of the wall confinement is smaller. We also notice that when $D_{o / i, \max }$ occurs, the outer droplet deviates from an ellipsoidal shape while the inner droplet always exhibits an ellipsoidal shape for all the confinement ratios considered (see side views in Fig. 11(b)). In addition, the minimum deformation parameters $D_{o / i, m i n}$ are slightly affected by the confinement ratio, and for high confinement ratios, the outer droplet seems not 
(a)

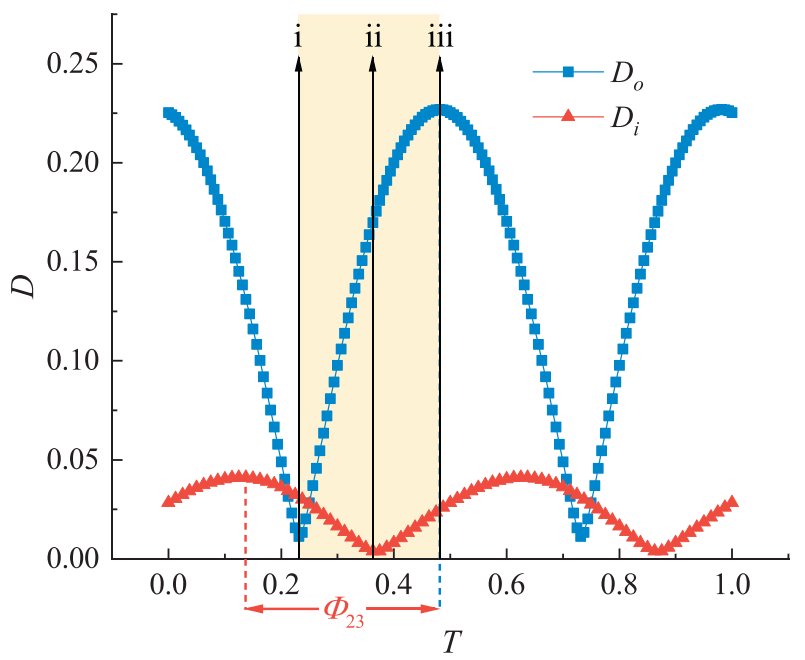

(b)

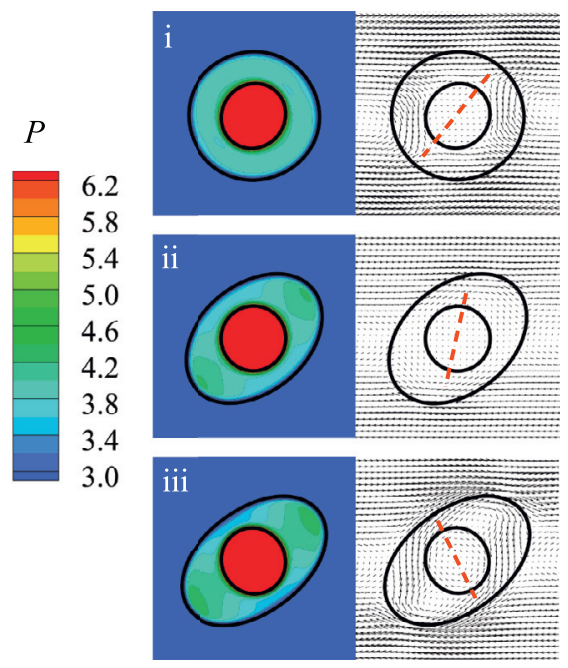

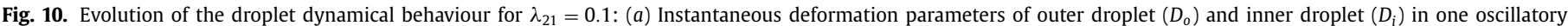

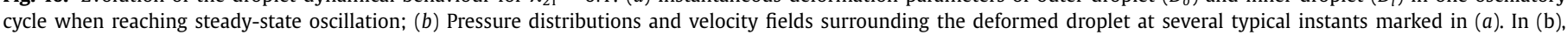

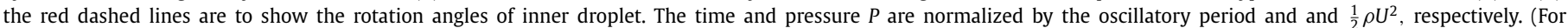
interpretation of the references to colour in this figure legend, the reader is referred to the web version of this article.)

(a)

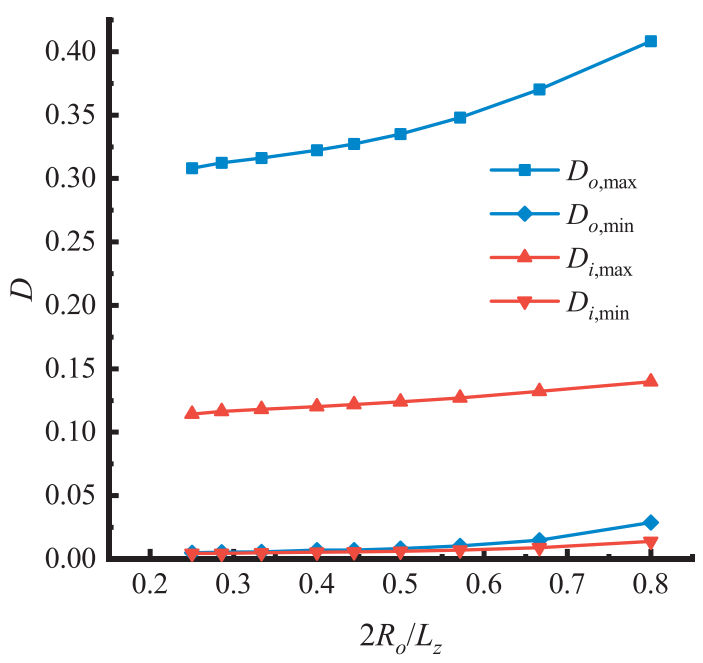

(b)

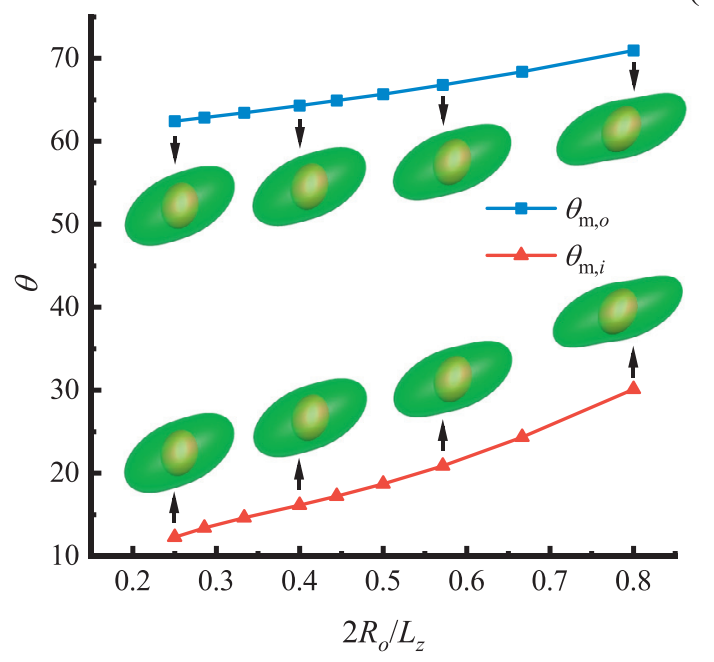

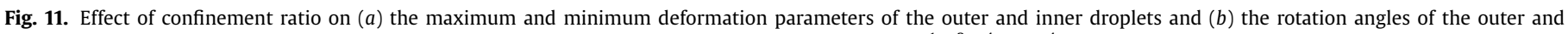
inner droplets when $D_{o / i, \max }$ are reached. A side view of the compound droplet is shown for $2 R_{o} / L_{z}=\frac{1}{4}, \frac{2}{5}, \frac{4}{7}$ and $\frac{4}{5}$ when $D_{o / i, \text { max }}$ are reached in $(b)$.

able to return to a truly spherical shape when $D_{o, \min }$ is reached. This is caused by the very small distance between the compound droplet and walls rather than by the shear flow, because the shear flow is extremely weak when $D_{0}$ reaches its minima (recall that the deformation of compound droplet almost synchronizes with the shear flow for the oscillatory period considered in this case). Fig. 11(b) shows the effect of the confinement ratio on the rotation angles $\theta_{\mathrm{m}, o / i}$ when $D_{o / i \text { max }}$ are reached. As the confinement ratio increases, the rotation angles $\theta_{\mathrm{m}, o}$ and $\theta_{\mathrm{m}, i}$ both increase, suggesting that both droplets orient more towards the flow direction. Hua et al. (2014) observed a similar behavior in the case of a compound droplet but in a simple shear flow. Based on the above results, one can conclude that the wall confinement could enhance the effect of the oscillatory shear. Comparison of the variations in the deformation parameters and rotation angles of both the droplets in Fig. 11(a,b) also reveals that the confinement ratio mainly contributes to promoting the deformation of the outer droplet and increasing the rotation of the inner droplet.
In the end, we point out that all the above discussion is based on a relatively low capillary number $(\mathrm{Ca}=0.25)$. In fact, for a relatively high $\mathrm{Ca}$, especially when approaching the critical value for droplet breakup, $D_{o, \max }$ may not exhibit a monotonic increase with the confinement ratio, which will be shown in Fig. 16.

\subsection{Effect of capillary number}

The capillary number $\mathrm{Ca}$, which represents the ratio of viscous to interfacial tension forces, serves as one of the most important parameters governing the droplet deformation and breakup in a shear flow. In this section, the effect of the capillary number is investigated for $R e=1, R_{i} / R_{0}=2 R_{o} / L_{z}=\frac{1}{2}, \lambda_{21}=\lambda_{31}=1$ and $T_{\text {osc }}=10 L_{x} / U$. The capillary number is limited to the range of 0.05 to 0.4625 so that the steady-state oscillatory deformation is finally attained for both inner and outer droplets.

Fig. 12 shows how the capillary number influences the maximum $\left(D_{i / o, \max }\right)$ and minimum $\left(D_{i / o, \min }\right)$ deformation parameters of 
(a)
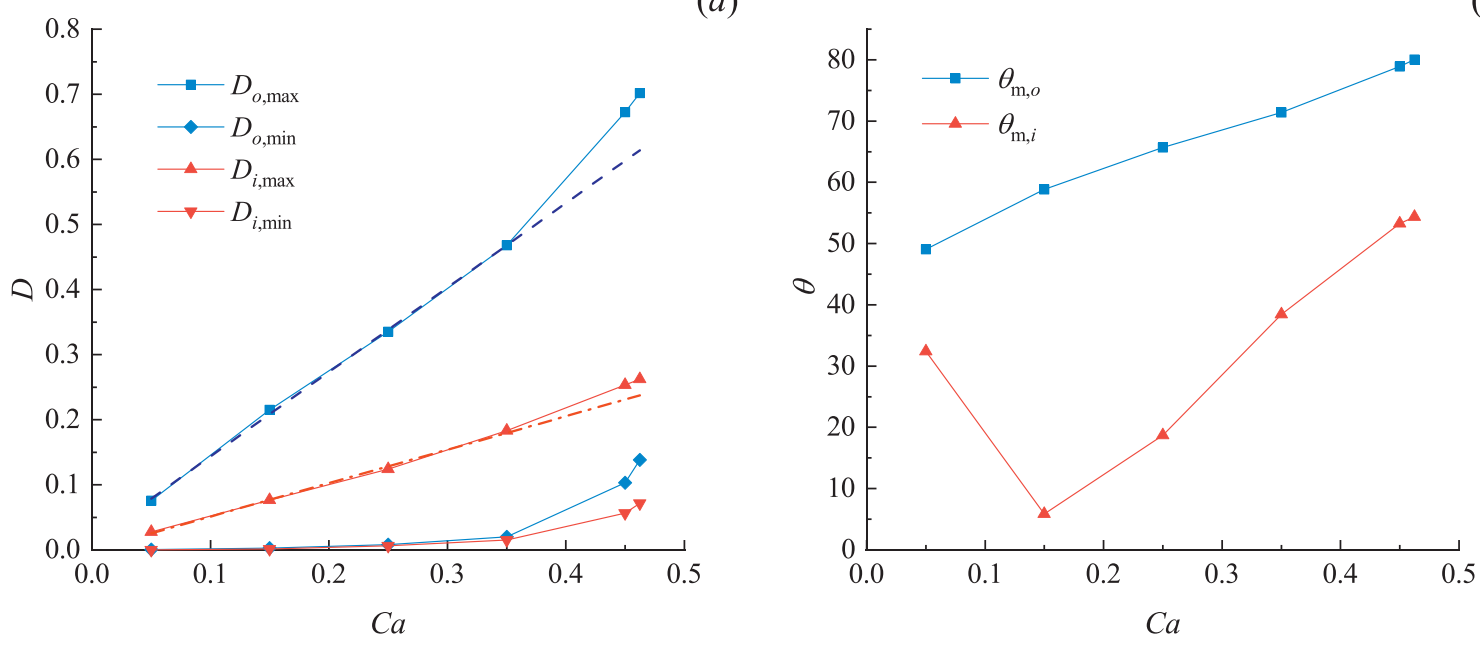

\section{$D_{o, \max }$}
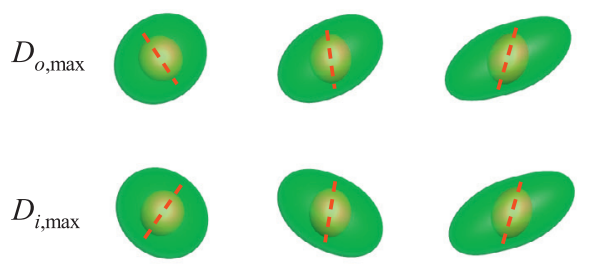

$C a=0.05$

$C a=0.15$
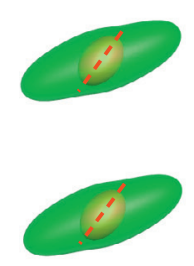

$C a=0.35$
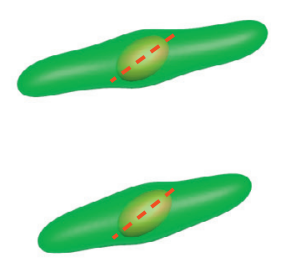

$C a=0.45$ (c)

(b)
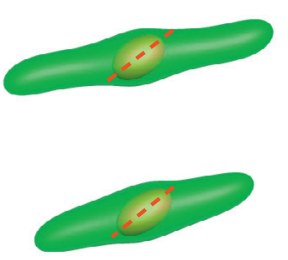

$C a=0.4625$

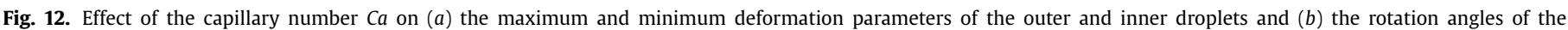

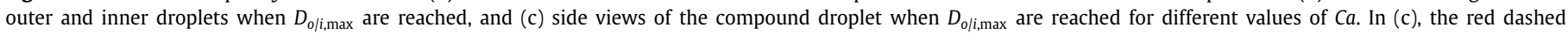

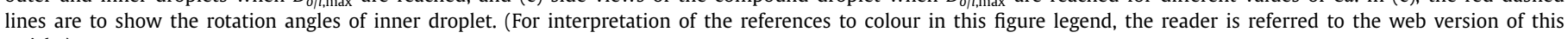
article.)

inner and outer droplets, and the rotation angles $\theta_{\mathrm{m}, i / o}$ and the corresponding droplet morphologies when $D_{i / 0, \max }$ are reached. As $\mathrm{Ca}$ increases, the outer droplet tends to be more stretched and inclined to the flow direction at the stage of its maximum deformation, and its shape gradually deviates from an ellipsoid (see the top row in Fig. 12(c)), which are also depicted by the growing $D_{o, \max }$ and $\theta_{\mathrm{m}, o}$ in Fig. $12(a, b)$. As for the inner droplet, the maximum deformation parameter $D_{i, \max }$ also increases with $\mathrm{Ca}$ but at a smaller rate compared with the outer droplet. Moreover, we can see in Fig. 12(a) that the maximum deformation parameters $D_{o / i \text { max }}$ vary approximately linearly with $C a$ up to $C a=0.35$ but start to deviate from the linearity at higher $\mathrm{Ca}$. This tendency is consistent with the experimental and numerical results concerning the deformation of a single-phase droplet in oscillatory shear flow (Wannaborworn et al., 2002) and in simple shear flow (Uijttewaal and Nijhof, 1995). When $\mathrm{Ca}>0.35$, the oscillatory deformation of the compound droplet becomes more complex, which is indicated by the sudden increase of $D_{o / i, \min }$ in Fig. 12(a). In fact, multipeaked oscillations of droplet deformation and orientation are observed for $\mathrm{Ca}>0.35$, which will be discussed later.

In Fig. $12(b)$, it is clearly observed that $\theta_{\mathrm{m}, i}$ first decreases and then increases with $\mathrm{Ca}$, different from the monotonic variation of $\theta_{\mathrm{m}, o}$. In addition, when $D_{i, \max }$ is reached, the inner and outer droplets incline in opposite directions for $\mathrm{Ca}=0.05$ and 0.15 but in the same directions for higher $\mathrm{Ca}$, as can be seen from the droplet morphologies shown in the bottom row of Fig. 12(c). This difference can be explained as follows. At low values of $\mathrm{Ca}$, the viscous shear force is so small that the behavior of the inner droplet is dominated by the pressure force arising from high pressure zones near two tips inside the outer droplet, and the dom- inant pressure force drives the inner droplet to rotate anticlockwise in the first half cycle when the outer droplet inclines to the right $\left(\Phi_{23} \approx-0.5\right.$ ). As the capillary number increases to 0.25 , the viscous shear force strengthens which causes the inner and outer droplets to almost synchronize with each other $\left(\Phi_{23} \approx 0\right)$. Upon further increasing $\mathrm{Ca}$, the deformation response of inner droplet would lag a little behind that of outer droplet, and both droplets exhibit complex non-linear behavior, which can be seen in Fig. 13.

Fig. 13(a) shows the instantaneous deformation parameters of both droplets and rotation angle of inner droplet in one steadystate oscillatory cycle for $\mathrm{Ca}=0.4625$, where five typical instants, i.e. (i)-(v), are selected for analysis, and the corresponding velocity fields surrounding the deformed droplet are plotted in Fig. 13(b). For the outer droplet, when reaching the maximum deformation, it deforms into a highly elongated shape (ii). Next, it contracts because of weakening shear flow and dominant interfacial tension. Since the outer droplet takes less time to contract than to stretch in one deformation cycle, the $D_{o}$ curve is no longer symmetric and its maximum point shifts to the right (see Fig. 13(a)). Then, the inner droplet reaches a local minimum deformation (iii), while the outer droplet is about to enter a rapid contraction stage, as indicated by the sharp decline of $D_{0}$ in Fig. 13(a). When the outer droplet undergoes a rapid contraction, the flow field formed around the inner droplet is something like an extensional flow which is capable of deforming and rotating the inner droplet further. As a result, the inner droplet reaches another local maximum deformation at the time of (iv). Afterwards, the outer droplet reaches its minimum deformation and the extensional-flow-like flow field disappears. Soon, the inner droplet relaxes to another local minimum deformation (v). During the relaxation of inner 


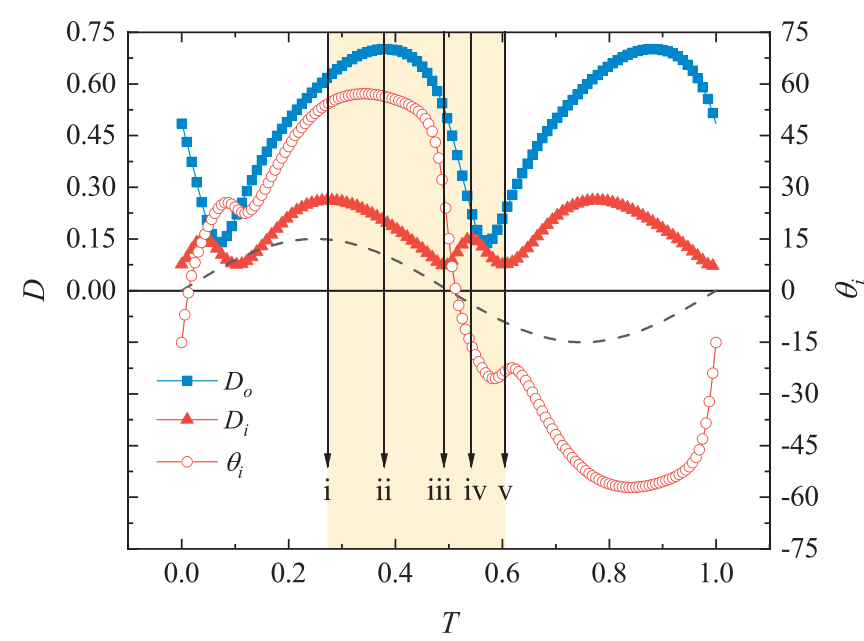

(b)
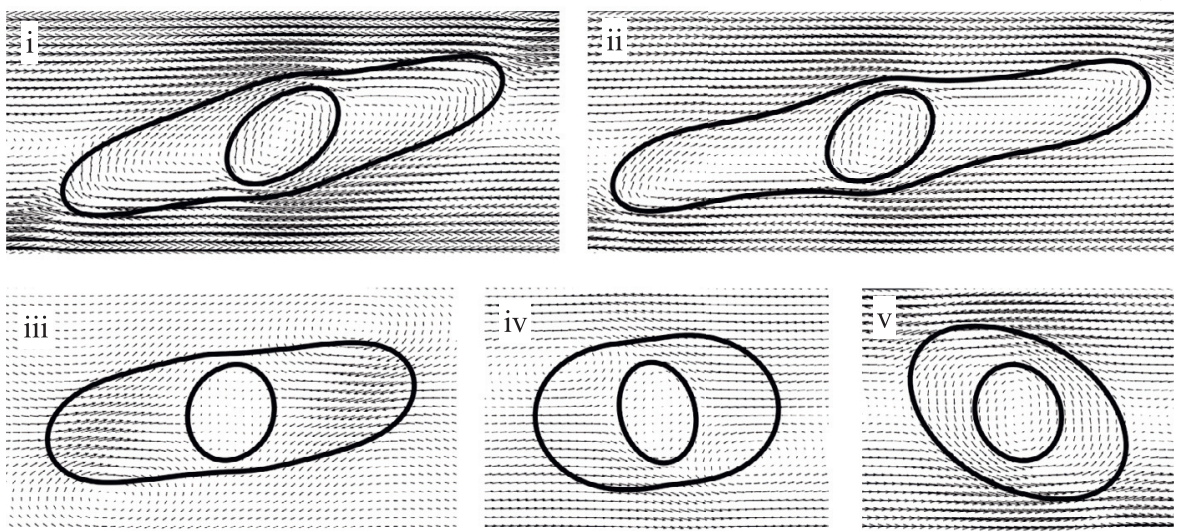

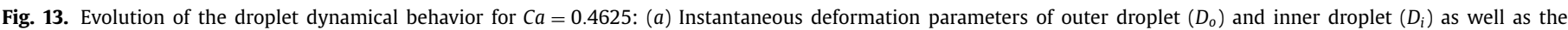

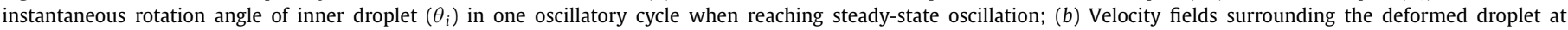
several typical instants marked in $(a)$. The time is normalized by the oscillatory period.

droplet, the instantaneous rotation angle $\theta_{i}$ is roughly a constant, and an anticlockwise vortical flow is formed interior to the outer droplet, which would deform the inner droplet subsequently. In addition, it is noticed in Fig. 13(b) that the tips of inner droplet are very close to the outer interfaces at the time of (i), leading to the behavior of inner droplet directly determined by the applied shear. So the inner droplet reaches its maximum deformation once $\dot{\gamma}_{\max }$ is reached, and then contracts with the decrease of $\dot{\gamma}$, regardless of the deformation of outer droplet.

As described above, the $D_{i}$ curve exhibits multiple local minima and maxima, i.e. the droplets undergoes multipeaked oscillations, at high values of $\mathrm{Ca}$, which is indicative of non-linearity. In fact, the behavior of multipeaked oscillations was also found by Guido et al. (2004), but they studied the behavior of a singlephase droplet subjected to an oscillatory shear flow. Finally, although there exist more complex responses at higher $\mathrm{Ca}$, the period of $\theta_{o / i}$ still equals to $T_{o s c}$ and the period of $D_{o / i}$ is only a half of $T_{\text {osc }}$, consistent with Guido et al. (2004) as well.

\section{Breakup of a compound droplet}

As the capillary number $\mathrm{Ca}$ increases, the droplet deformation increases until a critical value $C a_{c}$ is reached, above which the steady-state oscillatory deformation of the compound droplet cannot be maintained. Here, the $C a_{c}$ is defined as the lowest capillary number at which either droplet breaks up or undergoes an irre- versible change in topological structure. In our simulations, the bisection method is adopted to obtain $\mathrm{Ca}_{c}$, and the error of $\mathrm{Ca}_{c}$ is limited to $6.25 \times 10^{-3}$, which is small enough. It was experimentally and numerically found that the critical capillary number $\mathrm{Ca}_{c}$ is a function of the wall confinement for a single-phase droplet under simple shear (Janssen et al., 2010; Liu et al., 2018; Wang et al., 2017). Inspired by these works, we investigate the effect of the confinement ratio on $\mathrm{Ca}_{c}$ and the droplet breakup mode for a compound droplet under oscillatory shear, and the results are compared with those under simple shear to emphasize the role of oscillatory shear. For either oscillatory or simple shear, six different values of confinement ratio are considered, i.e. $2 R_{0} / L_{z}=\frac{1}{3}, \frac{2}{5}, \frac{1}{2}$, $\frac{4}{7}, \frac{2}{3}$ and $\frac{4}{5}$, and the other parameters are set to $R_{i} / R_{0}=\frac{1}{2}, R e=1$, $\lambda_{21}=\lambda_{31}=1$ and $T_{\text {osc }}=10 L_{x} / U$.

First, we consider a special case of $2 R_{0} / L_{z}=\frac{1}{2}$, in which the critical capillary number of droplet breakup is 0.46875 for oscillatory shear flow. Fig. 14 shows the snapshots of droplet breakup in the first two oscillatory cycles at $C a=0.46875$, where the time $T$ is normalized by the oscillatory period. It is seen that the inner and outer droplets successively reach their maximum deformations at $T_{1}$ and $T_{2}$ respectively during the first half cycle. This is similar to the case of $C a=0.4625$ shown in Fig. 13 , where the compound droplet eventually achieves steady-state oscillatory deformation. Then, as the external shear flow weakens, the outer droplet enters a rapid contraction stage and has a tendency of driving the inner droplet to approach another local maximum de- 


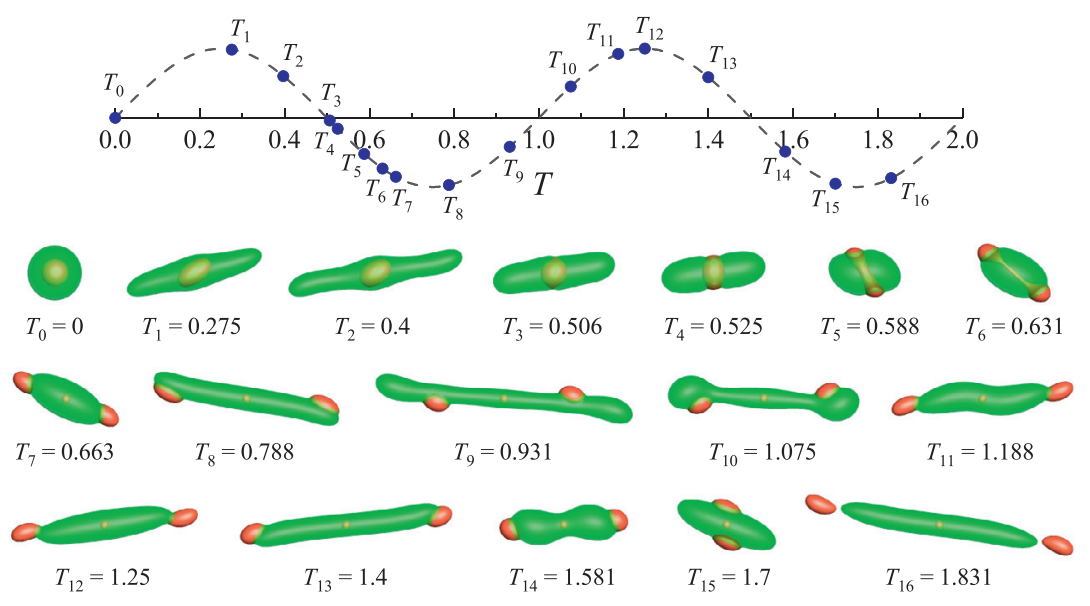

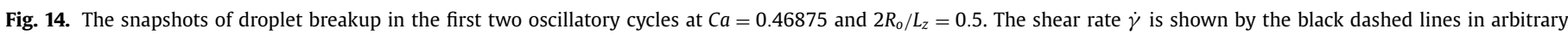
scale, on which 17 different instants are marked with the corresponding snapshots presented below. The time $T$ is normalized by the oscillatory period.

formation, like the process (iii)-(iv) shown in Fig. 13(a). However, soon after the inner droplet rotates anticlockwise, it touches the interface of the outer droplet at $T_{3}=0.506$. Subsequently, the inner droplet breaks through the surface of the outer droplet mainly due to interfacial tension, leading to partial exposure to the matrix fluid (see at $T_{4}$ and $T_{5}$ ). This means that the inner droplet will be affected by the reversed applied shear directly and by the interfacial tension between the matrix and inner droplet fluids. After that, as the reversed applied shear strengthens, the inner droplet is stretched longer and more inner droplet penetrates into the matrix fluid. Finally, the inner droplet breaks up into three parts: the smaller one staying in the center of outer droplet and the other two fragmented daughter droplets adhering to the tips of outer droplet (see at $T_{6}$ and $T_{7}$ ). As the time processes, the outer droplet becomes stretched and contracted with the oscillatory shear flow, exhibiting a variety of morphologies, and two daughter droplets of the core fluid keep sliding over the deformed outer droplet until separated at $T_{16}$.

As shown above, the breakup process of a compound droplet is full of diversity while it is endless. Therefore, the breakup mode is defined by the first breakup behavior of either inner or outer droplet. For $2 R_{0} / L_{z}=0.5$ in Fig. 14, the inner droplet is found to break into three parts when the first breakup occurs, and this mode is thus named as "inner ternary breakup". We then vary the confinement ratio to study its influence on $\mathrm{Ca}_{c}$ and breakup mode, and the relevant results are shown in Fig. 15. In this figure, the inset shows the droplet shapes when the inner droplet comes into contact with the outer interface and when the inner droplet just undergoes the first breakup. It can be observed that for a compound droplet under oscillatory shear, $\mathrm{Ca}_{c}$ first increases and then decreases with increasing confinement ratio, and the maximum value of $C a_{c}$ occurs at $2 R_{o} / L_{z}=0.4$. This is consistent with the variation of $D_{o, \max }$ at high $\mathrm{Ca}$, which is shown in Fig. 16. Unlike the results at low $\mathrm{Ca}$, e.g. $\mathrm{Ca}=0.25$, which are represented by the filled squares in Fig. 16 and extracted from Fig. 11(a), $D_{o \text { max }}$ does not exhibit a monotonic increase with the confinement ratio at $C a=0.45$ and 0.4625 . Within the range of steadystate oscillatory deformation, namely the region below the dashed line (note that the dashed line represents the threshold of $D_{0, \max }$, above which the breakup would occur), $D_{o, \max }$ increases gradually with the confinement ratio after an initial decline for $C a=0.45$ and 0.4625 , with their minimum values both at $2 R_{o} / L_{z}=0.4$. This means that the compound droplet is harder to be deformed, and thus a higher $C a_{c}$ is needed for droplet breakup, at $2 R_{o} / L_{z}=0.4$. In addition, it is seen from the insets that the compound droplet exhibits similar breakup behavior for $2 R_{0} / L_{z} \leqslant \frac{2}{3}$ : the inner droplet

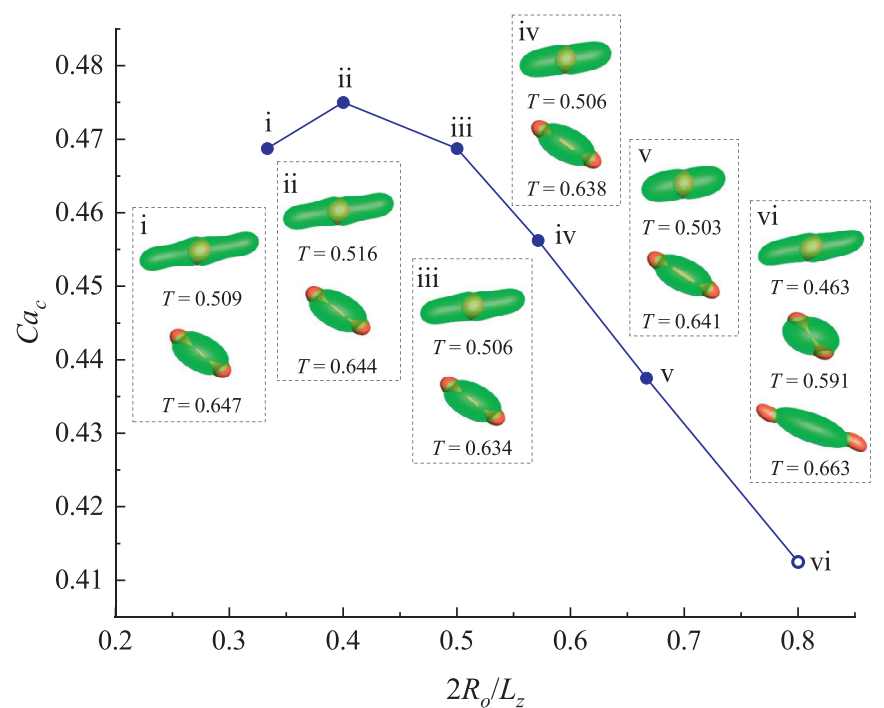

Fig. 15. Effect of the confinement ratio on the critical capillary number of droplet breakup. Each inset shows the droplet shapes and the corresponding times when the inner droplet comes into contact with the outer interface and when the inner droplet just undergoes the first breakup, where the time $T$ is normalized by the oscillatory period. The filled circles represent ternary breakup while the empty circle represents binary breakup.

comes into contact with the outer interface at $T \approx 0.5$ and breaks up at $T \approx 0.64$; the compound droplet undergoes the so-called inner ternary breakup. However, the deformation extent of the outer droplet when the contact occurs decreases with the increase of confinement ratio. This can be explained as follows: as the wall confinement increases, the outer droplet could better catch up with the oscillatory shear (see the discussion in Subsection 3.3), which enables the outer droplet to enter the rapid contraction stage earlier; as a result, the outer droplet has more time to contract until the contact occurs at $T \approx 0.5$. When the confinement ratio is increased to $2 R_{0} / L_{z}=0.8$, the inner droplet touches the outer interface and breaks up much earlier. More importantly, it is divided not into three parts but into two equal-sized parts. This breakup mode is termed as "inner binary breakup".

Next, we turn to the limiting case with infinite oscillatory period, namely simple shear flow, and obtain the values of $\mathrm{Ca}_{c}$ for varying confinement ratios, which are presented in Fig. 17(a). In this figure, the results of oscillatory shear flow in Fig. 15 are also shown for comparison. For both oscillatory and simple shear flows, 


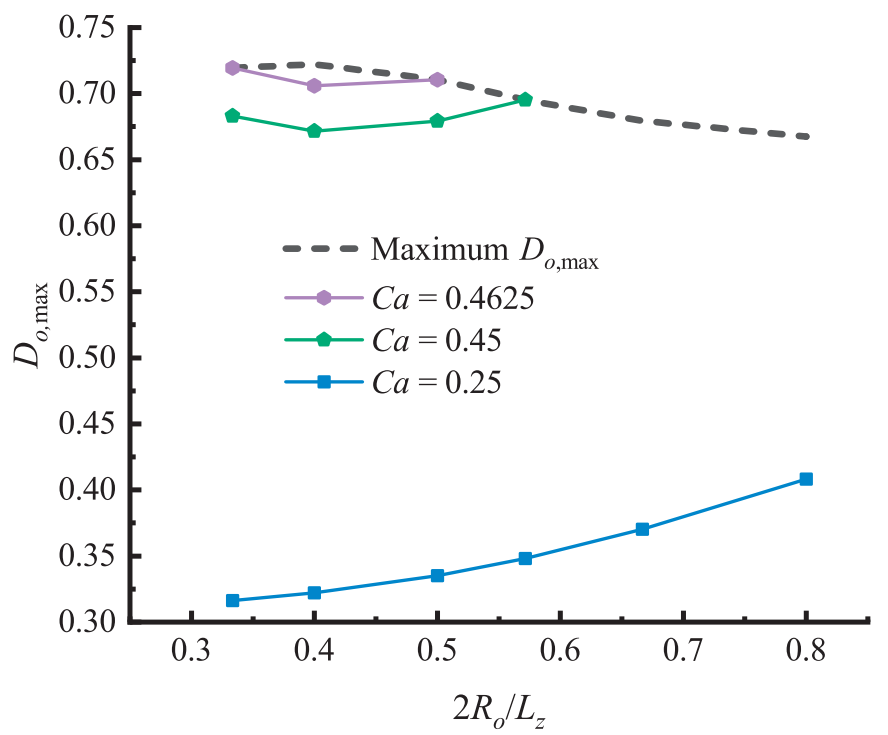

Fig. 16. Effect of the confinement ratio on $D_{o, \max }$ for several different values of $\mathrm{Ca}$. The black dashed line represents the threshold for $D_{o, \max }$, above which the droplet breakup would happen. the variations of $\mathrm{Ca}_{c}$ with confinement ratio exhibit the same trend, but the confinement ratios corresponding to the maximum value of $C a_{c}$ are somehow different: $2 R_{o} / L_{z}=0.4$ for the oscillatory shear flow and $2 R_{0} / L_{z}=0.5$ for the case of the simple shear flow. For a fixed confinement ratio, the critical capillary number for droplet breakup is always higher in the oscillatory shear flow than in the simple shear flow, which is consistent with the finding of Wannaborworn et al. (2002), who focused on the behavior of a single-phase droplet. This is easily understandable in view of the fact that in oscillatory shear flow, a compound droplet produces smaller deformation than in an equivalent steady shear flow, and thus a higher $\mathrm{Ca}$ is needed for droplet breakup.

Fig. 17(a) also shows that as the confinement ratio increases from $\frac{1}{3}$ to $\frac{4}{5}$, the droplet breakup mode undergoes the transition from the "outer ternary breakup" to the "inner ternary breakup" in simple shear flow. Again, we define the breakup mode through the first breakup behavior of either inner or outer droplet. To distinguish different modes of droplet breakup, we plot the snapshots of a compound droplet undergoing outer ternary breakup $\left(2 R_{0} / L_{z}=\frac{1}{3}\right.$ and $\left.C a_{c}=0.35\right)$ and inner ternary breakup $\left(2 R_{o} / L_{z}=\frac{4}{5}\right.$ and $C a_{c}=$ $0.3125)$ in Fig. $17(b)$, where the dimensionless time is defined as $\tau=\dot{\gamma} t$. At low confinement ratio, i.e. $2 R_{o} / L_{z}=\frac{1}{3}$, it is seen in (i)

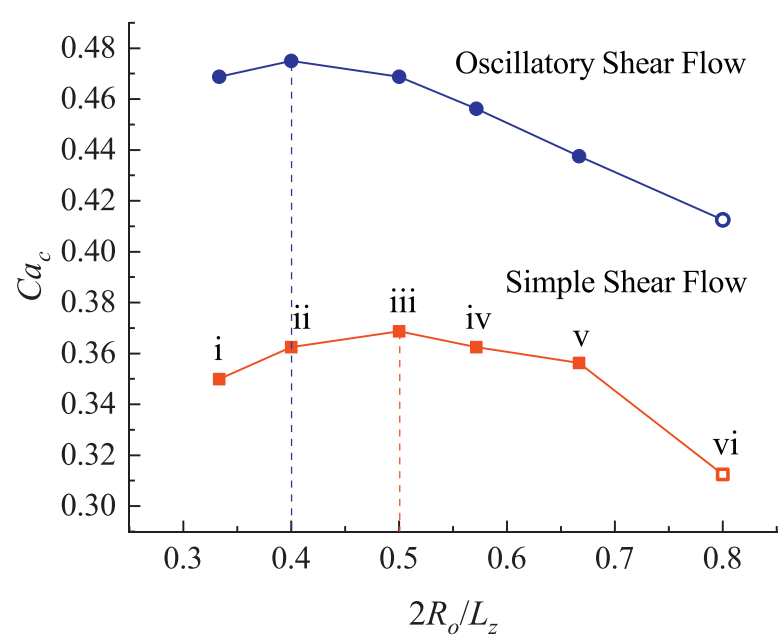

(a)

i

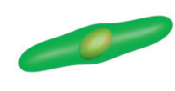

$\tau=123.05$

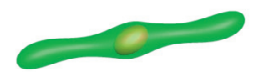

$\tau=137.84$

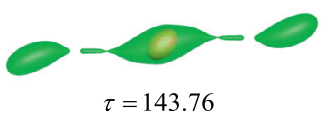

$\tau=143.76$

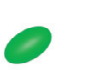

-

$\tau=146.72$

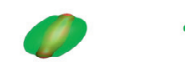

$\tau=152.63$ vi

(b)

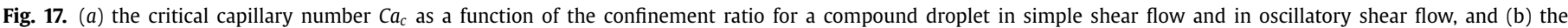

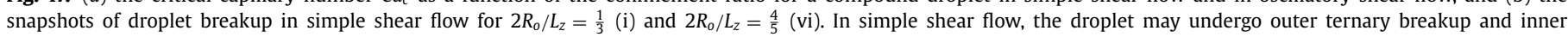
ternary breakup, which are represented by filled and empty squares, respectively. In (b), the dimensionless time is defined as $\tau=\dot{\gamma} t$. 
(a)
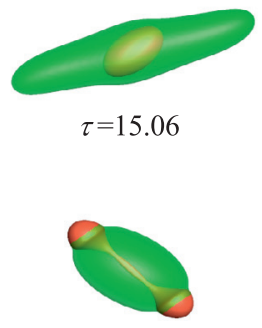

$\tau=34.57$

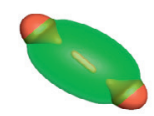

$\tau=34.75$

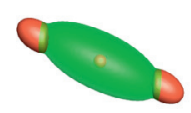

$\tau=36.29$

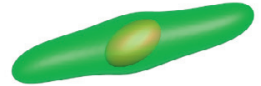

$\tau=28.03$

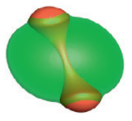

$\tau=57.37$

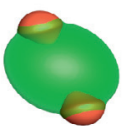

$\tau=58.02$

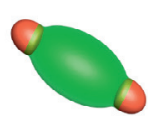

$\tau=61.28$ (b)

(c)
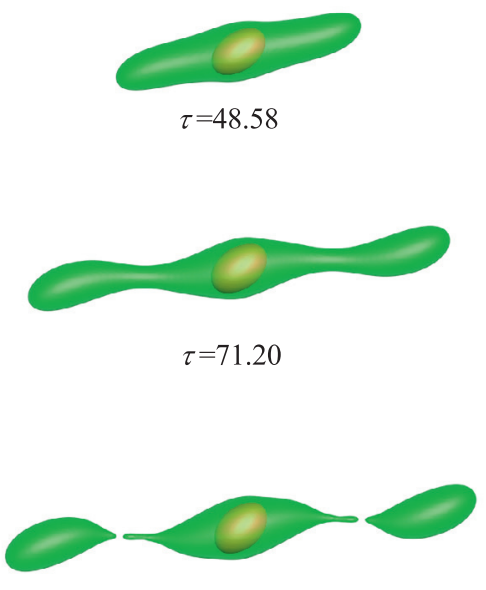

$\tau=74.24$

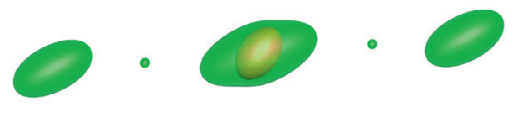

$\tau=75.75$

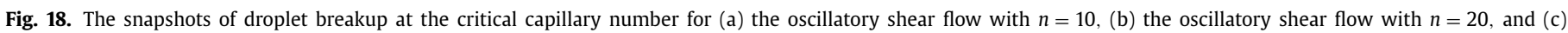
the simple shear flow. The confinement ratio is fixed at $2 R_{o} / L_{z}=1 / 2$, and the dimensionless time is defined as $\tau=\dot{\gamma} t$.

of Fig. 17(b) that the outer droplet first experiences a larger degree of elongation along the flow direction and its end parts start to develop into bulbous shapes with two necks formed on both sides of the inner droplet $(\tau=137.84)$. Then the two bulbous ends move away from the center and the necks are both stretched into thin threads until the first breakup occurs. The fragments generated upon the first breakup are three daughter droplets, including two single-phase droplets and one compound droplet with long threads in the center $(\tau=143.76)$. Quickly, both threads separate from the center compound droplet, leading to the formation of two minor satellites $(\tau=146.72)$. After that, the compound droplet continues to deform and rotate $(\tau=152.63)$, and eventually the inner droplet breaks up through the inner breakup mode as shown below. By contrast, at the high confinement ratio, i.e. $2 R_{o} / L_{z}=\frac{4}{5}$ (see (vi) in Fig. 17(b)), the compound droplet deforms and rotates much faster, so the inner droplet comes into contact with the outer interface as early as $\tau=4.61$. Then, the inner droplet partially penetrates into the matrix fluid, and it is divided into three parts under the action of steady shear flow at $\tau=9.36$. Next, the two side droplets of inner fluid slide over the outer droplet and towards its two ends, and finally they are separated from the center compound droplet $(\tau=13.84)$. It should be emphasized that the inner ternary breakup under simple shear is a direct result of the applied shear flow, distinct from the one under oscillatory shear, where the breakup arises from the reversing shear flow in the second half cycle of the first oscillatory period. Our results in both oscillatory and simple shear flows also show that the inner droplet is easier to break up as the confinement ratio increases. Compared with other values of confinement ratio, the breakup of the inner droplet occurs much earlier at $2 R_{o} / L_{z}=\frac{4}{5}$, although its corresponding $C a_{c}$ is lower.

Finally, a few more simulations are conducted to investigate how the oscillatory period influences the critical capillary number $C a_{c}$ and the breakup mode. In addition to the simple shear flow with $n \rightarrow \infty$, three different values of $n$, i.e. $n=1,10$ and 20 , are considered for a particular confinement ratio of $2 R_{0} / L_{z}=1 / 2$.
Table 1

Effect of the oscillatory period (expressed as $n$ ) on the critical capillary number and breakup mode. The inner ternary breakup, inner binary breakup and outer ternary breakup are denoted as ITB, IBB and OTB, respectively. ' - ' represents no breakup observed even for $C a$ up to 4 .

\begin{tabular}{lllll}
\hline$n$ & 1 & 10 & 20 & $\infty$ (simple shear) \\
\hline $\mathrm{Ca}_{c}$ & - & 0.46875 & 0.425 & 0.36875 \\
Breakup mode & - & ITB & IBB & OTB \\
\hline
\end{tabular}

The other parameters are fixed at $R_{0} / R_{i}=1 / 2, \lambda_{21}=\lambda_{31}=1$ and $R e=1$. Table 1 shows the values of $C a_{c}$ for different values of $n$. It is seen that for $n=1$, the breakup does not happen even for $\mathrm{Ca}$ up to 4 (the corresponding kinematic viscosity is 0.4 ). Note that a higher $\mathrm{Ca}$ is reachable but not recommended because as the viscosity (or $\mathrm{Ca}$ ) increases so does the Knudsen number, leading to a violation of the continuous medium hypothesis of fluid (Chen and Doolen, 1998). Thus, we have not reported the critical capillary number for $n=1$. Increasing the oscillatory period is found to decrease the value of critical capillary number. This indicates that the compound droplet tends to break up more easily as the oscillatory period increases, which is consistent with the predictions of the maximum deformation presented in Section 3.1. In addition, we notice that the compound droplet exhibits different breakup modes for these cases. Specifically, as the oscillatory period increases, the droplet undergoes the transition from inner ternary breakup to inner binary breakup, and finally to outer ternary breakup in simple shear flow $(n \rightarrow \infty)$, which is shown in Fig. 18. For $n=20$, at the onset of breakup, the outer droplet exhibits a smaller deformation than $n=10$ and the inner droplet appears to be saddle-shaped with its two bulbous ends directly in contact with the matrix fluid. Then, under the influence of the reversing shear flow, the neck of the inner droplet is stretched into a thin thread and eventually breaks up into two fragments. The generated two daughter droplets move quickly towards the tips of 
the outer droplet. In contrast, for the simple shear flow, the inner droplet in the first breakup process is less influenced by the external shear flow (see Fig. 18(c)); the outer droplet undergoes an outer ternary breakup, with its behavior similar to the simple shear flow with the confinement ratio $2 R_{o} / L_{z}=\frac{1}{3}$ (see (i) in Fig. $17(b)$ ). Based on the above discussion, one can conclude that the breakup extent of the inner droplet decreases with the oscillatory period, experiencing the transition from ternary breakup to binary breakup, and finally to no breakup in simple shear flow.

\section{Conclusions}

In this work, a three-phase lattice Boltzmann model is applied to study the dynamic behavior of a compound droplet when subjected to a three-dimensional oscillatory shear flow. Initially, a spherical compound droplet is placed in the center of two parallel walls, which move with equal sinusoidally oscillating speed but in opposite directions. At low capillary numbers, it is observed that the compound droplet deforms and eventually reaches a steadystate oscillatory condition, where the deformation parameters and rotation angles of inner and outer droplets no longer change from one oscillatory cycle to the next. In particular, the effects of oscillatory period, viscosity ratios, confinement ratio and capillary number $(\mathrm{Ca})$ on the deformation dynamics of the compound droplet, when the steady-state oscillatory conditions are reached, are systematically examined. Numerical results show that, as the oscillatory period increases, the maximum deformation parameters of inner and outer droplets gradually approaches the steady-state values of the corresponding simple shear flow, and the compound droplet becomes more synchronous with the applied shear. Also, the inner droplet is found to rotate counterintuitively in a direction opposite to the rotation of the outer droplet during the reversal of the shear flow, which is attributed to the dominant torque arising from the high pressure near the tips inside the outer droplet. The compound droplet often undergoes larger deformation when the outer or inner droplet is less viscous, which on the other hand mildly increases the synchronization between compound droplet and applied shear but decreases the synchronization between inner and outer droplets. Increasing the confinement ratio not only makes both constituent droplets more synchronous with the applied shear, but also promotes the extent of deformation. It is found that the maximum deformation parameters of inner and outer droplets are almost linearly proportional to $\mathrm{Ca}$ for $\mathrm{Ca} \leq 0.35$, but deviate from the linear relationship for higher $\mathrm{Ca}$. Moreover, multipeaked oscillations are observed for the deformation of inner droplet at high values of $\mathrm{Ca}$, wherein a secondary peak can be attributed to the extensional flow arising from the rapid contraction of outer droplet. When the capillary number is increased to a critical value, known as the critical capillary number $\left(\mathrm{Ca}_{c}\right)$, either inner or outer droplet would break up into different parts. The variation of $\mathrm{Ca}_{c}$ for different confinement ratios is investigated, and the results are compared with those observed in the case of simple shear flow. In the oscillatory shear flow, $\mathrm{Ca}_{c}$ first increases and then decreases with increasing confinement ratio, and the maximum $\mathrm{Ca}_{c}$ occurs at the confinement ratio of 0.4. Although in the simple shear, $\mathrm{Ca}_{c}$ exhibits a similar trend, the maximum $\mathrm{Ca}_{c}$ is reached at the confinement ratio of 0.5 . For all the confinement ratios considered, the value of $\mathrm{Ca}_{c}$ is always higher in the case of oscillatory shear. In addition, as the confinement ratio increases, the droplet breakup mode undergoes the transition from the inner ternary breakup to the inner binary breakup in the case of oscillatory shear, while it undergoes the transition from the outer ternary breakup to the inner ternary breakup in the case of simple shear flow. Finally, the droplet breakup is studied for varying oscillatory period. We find that increasing the oscillatory period decreases the value of $\mathrm{Ca}_{c}$ and the droplet breakup undergoes the transition from inner ternary breakup to inner binary breakup, and finally to outer ternary breakup.

\section{Declaration of Competing Interest}

The authors declare that they have no known competing financial interests or personal relationships that could have appeared to influence the work reported in this paper.

\section{CRediT authorship contribution statement}

Haihu Liu: Conceptualization, Methodology, Formal analysis, Resources, Data curation, Writing - review \& editing, Supervision, Project administration, Funding acquisition. Yang Lu: Methodology, Software, Validation, Formal analysis, Investigation, Writing original draft, Visualization. Sheng Li: Formal analysis, Investigation, Writing - original draft, Visualization. Yuan Yu: Methodology, Software. Kirti Chandra Sahu: Formal analysis, Writing - review \& editing.

\section{Acknowledgments}

This work is supported by the National Natural Science Foundation of China (No. 51876170), the Natural Science Basic Research Plan in Shaanxi Province of China (No. 2019JM-343) and the National Key Research and Development Project of China (No. 2016YFB0200902).

\section{References}

Aston, J.G., 1972. Gas-filled hollow drops in aerosols. J.Colloid Interface Sci. 38, 547-553.

Balla, M., Tripathi, M.K., Sahu, K.C., 2020. A numerical study of a hollow water droplet falling in air. Theor. Comput. Fluid Dyn. 34, 133-144.

Borthakur, M.P., Biswas, G., Bandyopadhyay, D., 2018. Dynamics of deformation and pinch-off of a migrating compound droplet in a tube. Phys. Rev. E 97, 043112.

Cavallo, R., Guido, S., Simeone, M., 2003. Drop deformation under small-amplitude oscillatory shear flow. Rheol. Acta 42, 1-9.

Che, Z., Yap, Y.F., Wang, T., 2018. Flow structure of compound droplets moving in microchannels. Phys. Fluids 30, 012114.

Chen, S., Doolen, G.D., 1998. Lattice Boltzmann method for fluid flows. Annu. Rev. Fluid Mech. 30 (1), 329-364.

Chen, H., Li, J., Shum, H.C., Stone, H.A., Weitz, D.A., 2011. Breakup of double emulsions in constrictions. Soft Matter 7, 2345-2347.

Chen, H., Zhao, Y., Li, J., Guo, M., Wan, J., Weitz, D.A., Stone, H.A., 2011. Reactions in double emulsions by flow-controlled coalescence of encapsulated drops. Lab Chip 11, 2312-2315.

Chen, Y., Liu, X., Shi, M., 2013. Hydrodynamics of double emulsion droplet in shear flow. Appl. Phys. Lett. 102, 051609.

Chen, Y., Liu, X., Zhang, C., Zhao, Y., 2015. Enhancing and suppressing effects of an inner droplet on deformation of a double emulsion droplet under shear. Lab Chip 15, 1255-1261.

Chen, Y., Liu, X., Zhao, Y., 2015. Deformation dynamics of double emulsion droplet under shear. Appl. Phys. Lett. 106, 141601.

Deka, H., Biswas, G., Sahu, K.C., Kulkarni, Y., Dalal, A., 2019. Coalescence dynamics of a compound drop on a deep liquid pool. J. Fluid Mech. 866, R21-11.

Farutin, A., Misbah, C., 2012. Rheology of vesicle suspensions under combined steady and oscillating shear flows. J. Fluid Mech. 700, 362-381.

Gao, P., Feng, J.J., 2011. Spreading and breakup of a compound drop on a partially wetting substrate. J. Fluid Mech. 682, 415-433.

Guido, S., Grosso, M., Maffettone, P.L., 2004. Newtonian drop in a newtonian matrix subjected to large amplitude oscillatory shear flows. Rheol. Acta 43, 575-583.

Gunstensen, A.K., Rothman, D.H., Zaleski, S., Zanetti, G., 1991. Lattice Boltzmann model of immiscible fluids. Phys. Rev. A 43 (8), 4320-4327.

Guo, Z., Shu, C., 2013. Lattice Boltzmann method and its applications in engineering. World Scientific Publishing Co. Pte. Ltd.

Guo, Z., Zheng, C., Shi, B., 2002. Discrete lattice effects on the forcing term in the lattice Boltzmann method. Phys. Rev. E 65, 046308.

Halliday, I., Hollis, A.P., Care, C.M., 2007. Lattice Boltzmann algorithm for continuum multicomponent flow. Phys. Rev. E 76, 026708.

Halliday, I., Law, R., Care, C.M., Hollis, A., 2006. Improved simulation of drop dynamics in a shear flow at low Reynolds and capillary number. Phys. Rev. E 73 (5), 056708.

Hua, H., Shin, J., Kim, J., 2014. Dynamics of a compound droplet in shear flow. Int. J. Heat Fluid Flow 50, 63-71.

Janpaen, V., Niamlang, S., Lerdwijitjarud, W., Sirivat, A., 2009. Oscillatory shear induced droplet deformation and breakup in immiscible polymer blends. Phys. Fluids 21, 063102. 
Janssen, P.J.A., Vananroye, A., Puyvelde, P.V., Moldenaers, P., Anderson, P.D., 2010. Generalized behavior of the breakup of viscous drops in confinements. J. Rheol. 54, 1047-1060.

Kim, S.-H., Shum, H.C., Kim, J.W., Cho, J.-C., Weitz, D.A., 2011. Multiple polymersomes for programmed release of multiple components. J. Am. Chem. Soc. 133, 15165-15171.

Krüger, T., Kusumaatmaja, H., Kuzmin, A., Shardt, O., Silva, G., Viggen, E.M., 2017. The lattice Boltzmann method. Springer International Publishing AG Switzerland.

Latva-Kokko, M., Rothman, D.H., 2005. Diffusion properties of gradient-based lattice boltzmann models of immiscible fluids. Phys. Rev. E 71, 056702.

Leclaire, S., Reggio, M., Trépanier, J.-Y., 2013. Progress and investigation on lattice Boltzmann modeling of multiple immiscible fluids or components with variable density and viscosity ratios. J. Comput. Phys. 246, 318-342.

Lee, D., Weitz, D.A., 2008. Double emulsion-templated nanoparticle colloidosomes with selective permeability. Adv. Mater. 20, 3498-3503.

Li, J., Chen, H., Stone, H.A., 2011. Breakup of double emulsion droplets in a tapered nozzle. Langmuir 27, 4324-4327.

Liu, H., Ba, Y., Wu, L., Li, Z., Xi, G., Zhang, Y., 2018. A hybrid lattice Boltzmann and finite difference method for droplet dynamics with insoluble surfactants. J. Fluid Mech. 837, 381-412.

Liu, H., Valocchi, A.J., Kang, Q., 2012. Three-dimensional lattice Boltzmann model for immiscible two-phase flow simulations. Phys. Rev. E 85, 046309.

Luo, Z.Y., He, L., Bai, B.F., 2015. Deformation of spherical compound capsules in simple shear flow. J. Fluid Mech. 775, 77-104.

Matsunaga, D., Imai, Y., Yamaguchi, T., Ishikawa, T., 2015. Deformation of a spherical capsule under oscillating shear flow. J. Fluid Mech. 762, 288-301.

Oguz, H.N., Sadhal, S.S., 1987. Growth and collapse of translating compound multiphase drops: analysis of fluid mechanics and heat transfer. J. Fluid Mech. 179, 105-136.

Oldroyd, J.G., 1953. The elastic and viscous properties of emulsions and suspensions. Proc. R. Soc. A 218, 122-132.

Qu, X., Wang, Y., 2012. Dynamics of concentric and eccentric compound droplets suspended in extensional flows. Phys. Fluids 24, 123302.

Sadhal, S.S., Oguz, H.N., 1985. Stokes flow past compound multiphase drops: the case of completely engulfed drops/bubbles. J. Fluid Mech. 160, 511-529.

Shah, R.K., Shum, H.C., Rowat, A.C., Lee, D., Agresti, J.J., Utada, A.S., Chu, L.-Y., Kim, J.-W., Fernandez-Nieves, A., Martinez, C.J., Weitz, D.A., 2008. Designer emulsions using microfluidics. Mater. Today 11, 18-27.

Smith, K.A., Ottino, J.M., Olvera de la Cruz, M., 2004. Encapsulated drop breakup in shear flow. Phys. Rev. Lett. 93, 204501.

Song, Y., Xu, J., Yang, Y., 2010. Stokes flow past a compound drop in a circular tube. Phys. Fluids 22, 072003.

Spencer, T.J., Halliday, I., Care, C.M., 2010. Lattice Boltzmann equation method for multiple immiscible continuum fluids. Phys. Rev. E 82, 066701.

Stone, H.A., Leal, L.G., 1990. Breakup of concentric double emulsion droplets in linear flows. J. Fluid Mech. 211, 123-156.
Succi, S., 2001. The lattice Boltzmann equation: For fluid dynamics and beyond. Oxford University Press.

Tao, J., Song, X., Liu, J., Wang, J., 2013. Microfluidic rheology of the multiple-emulsion globule transiting in a contraction tube through a boundary element method. Chem. Eng. Sci. 97, 328-336.

Taylor, G.I., 1934. The formation of emulsions in definable fields of flow. Proc. R. Soc. Lond. A 146, 501-523.

Tiribocchi, A., Montessori, A., Aime, S., Milani, M., Lauricella, M., Succi, S., Weitz, D., 2020. Novel nonequilibrium steady states in multiple emulsions featured. Phys. Fluids 32, 017102.

Uijttewaal, W.S.J., Nijhof, E.J., 1995. The motion of a droplet subjected to linear shear flow including the presence of a plane wall. J. Fluid Mech. 302, 45-63.

Utada, A.S., Lorenceau, E., Link, D.R., Kaplan, P.D., Stone, H.A., Weitz, D.A., 2005 Monodisperse double emulsions generated from a microcapillary device. Science 308, 537-541.

Vu, T.V., Vu, L.V., Pham, B.D., Luu, Q.H., 2018. Numerical investigation of dynamic behavior of a compound drop in shear flow. J. Mech. Sci. Technol. 32, 2111-2117.

Vu, T.-V., Vu, T.V., Bui, D.T., 2019. Numerical study of deformation and breakup of a multi-core compound droplet in simple shear flow. Intl J. Heat Mass Tran. 131, 1083-1094.

Wang, J., Li, X., Wang, X., Guan, J., 2014. Possible oriented transition of multiple-emulsion globules with asymmetric internal structures in a microfluidic constriction. Phys. Rev. E 89, 052302.

Wang, J., Liu, J., Han, J. Guan, J., 2013. Effects of complex internal structures on rheology of multiple emulsions particles in $2 \mathrm{~d}$ from a boundary integral method. Phys. Rev. Lett. 110, 066001.

Wang, N., Liu, H., Zhang, C., 2017. Deformation and breakup of a confined droplet in shear flows with power-law rheology. J. Rheol. 61, 741-758.

Wannaborworn, S., Mackley, M.R., Renardy, Y., 2002. Experimental observation and matching numerical simulation for the deformation and breakup of immiscible drops in oscillatory shear. J. Rheol. 46, 1279-1293.

Yu, Y., Liang, D., Liu, H., 2019. Lattice Boltzmann simulation of immiscible threephase flows with contact-line dynamics. Phys. Rev. E 99, 013308.

Yu, Y., Liu, H., Liang, D., Zhang, Y., 2019. A versatile lattice Boltzmann model for immiscible ternary fluid flows. Phys. Fluids 31, 012108.

Zhao, M., Bagchi, P., 2011. Dynamics of microcapsules in oscillating shear flow. Phys. Fluids 23, 111901.

Zhou, C., Yue, P., Feng, J.J., 2008. Deformation of a compound drop through a contraction in a pressure-driven pipe flow. Int. J. Multiphase Flow 34, 102-109.

Zhu, L., Gallaire, F., 2017. Bifurcation dynamics of a particle-encapsulating droplet in shear flow. Phys. Rev. Lett. 119 (6), 064502.

Zhu, L., Rabault, J., Brandt, L., 2015. The dynamics of a capsule in a wall-bounded oscillating shear flow. Phys. Fluids 27, 071902.

Zou, Q., He, X., 1997. On pressure and velocity boundary conditions for the lattice Boltzmann BGK model. Phys. Fluids 9, 1591-1598. 